HUPD-0110

hep-th/0107033

\title{
Noncommutative Cohomological Field Theory and GMS soliton
}

\author{
Tomomi Ishikawa 月, Shin-Ichiro Kuroki and Akifumi Sako \\ Graduate School of Science, Hiroshima University, \\ 1-3-1 Kagamiyama, Higashi-Hiroshima 739-8526, Japan
}

\begin{abstract}
We show that it is possible to construct a quantum field theory that is invariant under the translation of the noncommutative parameter $\theta_{\mu \nu}$. This is realized in a noncommutative cohomological field theory. As an example, a noncommutative cohomological scalar field theory is constructed, and its partition function is calculated. The partition function is the Euler number of Gopakumar, Minwalla and Strominger (GMS) soliton space.
\end{abstract}

\footnotetext{
${ }^{*}$ E-mail address: tomomi@theo.phys.sci.hiroshima-u.ac.jp

${ }^{\dagger}$ E-mail address: kuroki@theo.phys.sci.hiroshima-u.ac.jp

${ }^{\ddagger}$ E-mail address: sako@math.sci.hiroshima-u.ac.jp
} 


\section{Introduction}

Recently noncommutative geometry and noncommutative field theory revived in the string theory 20. There are some correspondences between commutative and noncommutative geometry. For example in the gauge theory, it was shown that the commutative theory with some background field is equivalent to the noncommutative theory 24 ). After that, a few kinds of nontrivial soliton in the noncommutative space are discovered. In the gauge theory, $U(1)$ instanton solution is discovered by Nekrasov and Schwartz 21), and in the scalar theory a nontrivial solution is discovered by Gopakumar, Minwalla and Strominger 14), which is called GMS soliton. There is no corresponding solution in the commutative space, i.e. the GMS soliton is a specific solution in the noncommutative space.

In the noncommutative space, it is difficult to define the length or metric. There are few examples, like a noncommutative torus case, derived the differential geometry, e.g., connection

and curvature $\mathbf{E}$. But in general noncommutative space, to define the Riemannian geometry is difficult. What we can to do is to classify the geometry to the extent of the algebric Ktheory. However, it is not enough to classify the noncommutative space from a point of view of differential topology. If there are some characteristic classes that do not varied under the shift from commutative to noncommutative space, then they are useful for the classification of spaces. For instance, in the poncommutative torus the Euler number is independent of the noncommutative parameter $\theta$ Bi). We expect that some other topological invariants would be extended to noncommutative space and independent of $\theta$.

The aim of this paper is to construct a quantum field theory that is invariant under the transformation of the noncommutative parameter $\theta^{\mu \nu}$. This parameter characterizes the noncommutativity of spaces as

$$
\left[x^{\mu}, x^{\nu}\right]=\frac{\theta^{\mu \nu}}{2 \pi i}
$$

where $x^{\mu}$ are the coordinates of the noncommutative space. Noncommutative parameter independence of the theory means that the partition function of the theory is independent of $\theta^{\mu \nu}$. Cohomological field theory is nominated as such a theory. We construct a cohomological field theory on the noncommutative space, and we show that it is actually a usual cohomological field theory in the $\theta^{\mu \nu}=0$ limit. This fact means that noncommutative space succeed to some geometric or topological information of commutative space.

Another purpose of this paper is to construct a concrete example of the invariants under the transformation of noncommutative parameter $\theta^{\mu \nu}$. The discovery of the GMS solution is one of the most important developments in recent work on noncommutative field theory. Therefore, we calculate the Euler number of the GMS soliton space as the example of $\theta^{\mu \nu}$ independent partition function. We will see the relation between the GMS soliton and commutative cohomological field theory.

Our example of the noncommutative cohomological field theory is a balanced scalar model that has two multiplets, the scalar and the vector. We consider the case that the potential is general degree polynomial of the scalar field. In the commutative space, the degree of the potential determines the structure of the vacuum. We will show that in the noncommutative space there is similar picture of vacuum structure. As a result of investigation, the correspondence between the commutative limit $\theta \rightarrow 0$ and the noncommutative limit $\theta \rightarrow \infty$ is obtained. Especially in the large $\theta$ limit, the potential term plays a dominant role, and there 
are the specific GMS solutions. A point of this paper is how we deal with the GMS solitons in quantum field theory.

This paper is organized as follows. In section II, we will construct the $\theta^{\mu \nu}$ invariant quantum field theory in general. In section III, we construct a scalar model as a simple example. Balanced topological theory will be used there. It is necessary to introduce potential in topological scalar field theory. Partition function is calculated in both limits, commutative and noncommutative. In section IV, we introduce the Morse theory on the noncommutative field theory, and show that the Euler number of GMS soliton space is well-defined. In the last section, we summarize and discuss our result.

\section{General formalism}

The aim of this section is to make a theory that is invariant under the shift of the noncommutative parameter $\theta_{\mu \nu}$. We discuss how we construct the cohomological field theory on the noncommutative space.

\section{II.i Cohomological field theory on noncommutative space}

The noncommutative parameter is defined in the commutation relation Eq.([.1). We introduce the infinitesimal rescaling operator $\delta_{s}$ as follows.

$$
\left(1-\delta_{s}\right)\left[x^{\mu}, x^{\nu}\right] \equiv\left[x^{\prime \mu}, x^{\prime \nu}\right]=\frac{\theta^{\mu \nu}-\delta \theta^{\mu \nu}}{2 \pi i} .
$$

This commutation relation is given by defining $\delta_{s}$ as

$$
\begin{aligned}
x^{\prime \mu} & =x^{\mu}-\delta_{s} x^{\mu}, \\
\delta_{s} x^{\mu} & =\left(\frac{1}{2} \delta \theta^{\mu \nu}\left(\theta^{-1}\right)_{\nu \rho}\right) x^{\rho} .
\end{aligned}
$$

This transformation corresponds to $x^{\prime \mu}=\sqrt{\theta} x^{\mu}$ in 14 . We denote the inverse matrix of the transformation (II.2) by

$$
J_{\rho}^{\mu} \equiv \delta_{\rho}^{\mu}+\frac{1}{2} \delta \theta^{\mu \nu}\left(\theta^{-1}\right)_{\nu \rho}
$$

Then, the integration measure and the differential operator are transformed into

$$
d x^{D}=\operatorname{det} \mathbf{J} d x^{\prime D}, \quad \frac{\partial}{\partial x^{\mu}}=\left(J^{-1}\right)_{\mu \nu} \frac{\partial}{\partial x^{\prime \nu}},
$$

where $\operatorname{det} \mathbf{J}$ is the Jacobian. By ([ᄑ.5) the Moyal product(see e.g.6) is shifted as

$$
\left(1-\delta_{s}\right)\left(*_{\theta}\right)=\delta_{s}\left(\exp \left(2 \pi i \overleftarrow{\partial}_{\mu}(\theta-\delta \theta)^{\mu \nu} \vec{\partial}_{\nu}\right)\right)=*_{\theta-\delta \theta}
$$

because $\delta_{s}\left(\overleftarrow{\partial}_{\mu} \theta^{\mu \nu} \vec{\partial}_{\nu}\right)=\overleftarrow{\partial}_{\mu} \delta \theta^{\mu \nu} \vec{\partial}_{\nu}$. Note that this transformation is just a rescaling of the coordinate, so that any action and its partition function are not changed under this transformation,

$$
\begin{aligned}
S_{\theta} & =\int d x^{D} \mathcal{L}\left(*_{\theta}, \partial_{\mu}\right) \\
& =\int \operatorname{det} \mathbf{J} d x^{\prime D} \mathcal{L}\left(*_{\theta-\delta \theta},\left(J^{-1}\right)^{\mu \nu} \frac{\partial}{\partial x^{\prime \nu}}\right)
\end{aligned}
$$


where $\mathcal{L}\left(*_{\theta}, \partial_{\mu}\right)$ is an explicit description to emphasis that the products of fields are the Moyal product and it contains derivative terms in the lagrangian. For convenience, we will often omit $*_{\theta}$ when we do not misunderstand. In the next step, we shift the noncommutative parameter.

$$
\theta \rightarrow \theta^{\prime}=\theta+\delta \theta
$$

This shift changes the action and the partition function in general, as follows

$$
S_{\theta^{\prime}}=\int \operatorname{det} \mathbf{J} d x^{\prime D} \mathcal{L}\left(*_{\theta},\left(J^{-1}\right)^{\mu \nu} \frac{\partial}{\partial x^{\prime \nu}}\right) .
$$

Compared with (II.7), the shift is regarded as a rescaling without the Moyal product.

Contrary, our purpose is to construct a field theory invariant under this shift. Immediately we expect the cohomological field theory would be an example since it is scale invariant theory $\mathrm{A}, \mathrm{A}, 26)$. Cohomological field theory is understood through several ways. Twisted SUSY is one of them, but noncommutative SUSY is not adverted here 1, 12, 15). Meanwhile, a geometrical point of view is closely studied in section IV. The Lagrangian of cohomological field theory is BRST-exact. We denote the BRST operator by $\hat{\delta}$, and generic bosonic fields by $\phi_{i}$, which are sections of some vector bundle $s_{a}\left(*_{\theta} \phi_{i}\right)$. BRST operator is defined as

$$
\begin{gathered}
\hat{\delta} \phi_{i}=\psi_{i}, \quad \hat{\delta} \psi_{i}=0, \\
\hat{\delta} \chi^{a}=H^{a}, \quad \hat{\delta} H^{a}=0,
\end{gathered}
$$

where $\phi_{i}$ and $H^{a}$ are bosonic, $\psi_{i}$ and $\chi^{a}$ are fermionic fields. Following the Mathai-Quillen formalism, the action of the cohomological field theory is written as

$$
\begin{aligned}
V & =\chi^{a}\left(i s_{a}+H_{a}\right), \\
S_{\theta} & =\int d x^{D} \mathcal{L}\left(*_{\theta}, \partial_{\mu}\right)=\int d x^{D} \hat{\delta} V .
\end{aligned}
$$

The partition function is defined by

$$
Z_{\theta}=\int \mathcal{D} \phi \mathcal{D} \psi \mathcal{D} \chi \mathcal{D} H \exp \left(-S_{\theta}\right)
$$

In the commutative space, the Mathai-Quillen formalism tells us that the partition function gives a representation of the Euler number of the space $\mathcal{M}=\left\{s_{a}^{-1}(0)\right\}$.

This partition function is invariant under an infinitesimal transformation which commute with the BRST transformation (II.10).

$$
\begin{aligned}
\hat{\delta} \delta^{\prime} & = \pm \delta^{\prime} \hat{\delta} \\
\delta^{\prime} Z_{\theta} & =\int \mathcal{D} \phi \mathcal{D} \psi \mathcal{D} \chi \mathcal{D} H \quad \delta^{\prime}\left(-\int d x^{D} \hat{\delta} V\right) \exp \left(-S_{\theta}\right) \\
& =\int \mathcal{D} \phi \mathcal{D} \psi \mathcal{D} \chi \mathcal{D} H \quad \hat{\delta}\left(-\int \delta^{\prime} V\right) \exp \left(-S_{\theta}\right)=0 .
\end{aligned}
$$

The vacuum expectation value(VEV) of any BRST-exact observable is zero. Note that the path integral measure is invariant under $\delta^{\prime}$ transformation since every field has only one supersymmetric partner and the Jacobian is totally canceled. 
We introduce the $\theta$-shift operator as

$$
\delta_{s} x_{\mu}=-\delta_{s} x_{\mu}, \quad \delta_{\theta} \theta_{\mu \nu}=\theta_{\mu \nu}+\delta \theta_{\mu \nu} .
$$

Generally, it is possible to define $\delta_{\theta}$ to commute with the BRST operator. Following (II.14), the partition function is invariant under this $\theta$-shift and it means that the Euler number of the space $\mathcal{M}$ is independent of the noncommutative parameter $\theta$.

In the end of this section, we list some general nature of this partition function. First, the Gaussian integral is formally defined and an exact result can be given by 1-loop calculation. Second, naively the commutative limit $(\theta \rightarrow 0)$ is given by removing potential terms without constant field, and $\theta \rightarrow \infty$ limit is given by omitting kinetic terms in the action, because the limit $\theta \rightarrow 0(\theta \rightarrow \infty)$ means $\mathbf{J} \rightarrow 0(\mathbf{J} \rightarrow \infty)$ in Eq.([I.9).

In the next section, as an example of noncommutative cohomological field theory, we investigate the balanced scalar model.

\section{Balanced Scalar model}

In this section, we study a concrete example of noncommutative cohomological field theory as $\theta$-shift invariant field theory. For simplicity, we use a real scalar model to construct a cohomological field theory. However, there is no nontrivial example under usual construction of cohomological field theory. Therefore, we introduce the balanced topological field theory 8.18. This theory is used in the investigation of the Vafa-Witten theory whose partition function is the sum of Euler number of the zero section space $\mathcal{M}$ without a sign, in general 10, 23, 25. When we calculate the Vafa-Witten theory, zero mode integration is not essential because of no ghost number anomaly. Contrary, in this paper, we carefully calculate the zero mode integration to compare the commutative limit with the large $\theta$ limit.

\section{III.i The action}

We construct the balanced scalar model. The theory is composed of bosonic scalar fields $\phi$ and $H$, fermionic scalar fields $\psi$ and $\chi$, bosonic vector fields $B_{\mu}$ and $H_{\mu}$, fermionic vector fields $\psi_{\mu}$ and $\chi_{\mu}$, and every field is Hermitian field. We give the BRST transformations (Fig.1) as

$$
\begin{aligned}
& \hat{\delta}_{+} \phi=\psi, \hat{\delta}_{-} \phi=\chi \\
& -\hat{\delta}_{-} \psi=\hat{\delta}_{+} \chi=H, \\
& \hat{\delta}_{+} \psi=\hat{\delta}_{-} \chi=\hat{\delta}_{+} H=\hat{\delta}_{-} H=0, \\
& \hat{\delta}_{+} B^{\mu}=\psi^{\mu}, \hat{\delta}_{-} B^{\mu}=\chi^{\mu}, \\
& -\hat{\delta}_{-} \psi^{\mu}=\hat{\delta}_{+} \chi^{\mu}=H^{\mu} \\
& \hat{\delta}_{+} \psi^{\mu}=\hat{\delta}_{-} \chi^{\mu}=\hat{\delta}_{+} H^{\mu}=\hat{\delta}_{-} H^{\mu}=0,
\end{aligned}
$$

where $\hat{\delta}_{+}$and $\hat{\delta}_{-}$satisfy the following relations,

$$
\hat{\delta}_{+}^{2}=\hat{\delta}_{-}^{2}=0, \quad \hat{\delta}_{+} \hat{\delta}_{-}+\hat{\delta}_{-} \hat{\delta}_{+}=0 .
$$



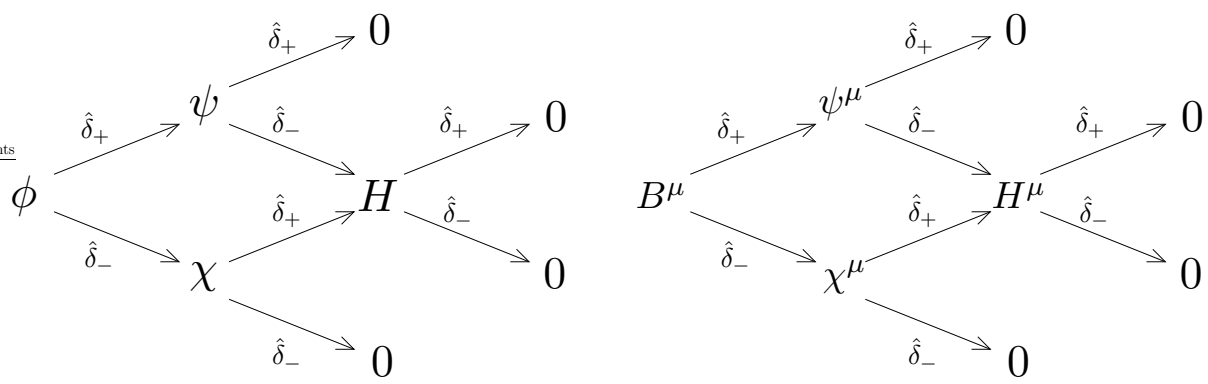

Figure 1: BRST multiplets in the balanced scalar model. $\delta_{+}\left(\delta_{-}\right)$carries ghost number $+1(-1)$.

The action of the balanced scalar model is

$$
S=\int d x^{D} \sqrt{g} \mathcal{L}
$$

where the Lagrangian $\mathcal{L}$ is given by an exact form with a ghost-number zero functional $\mathcal{F}$ as

$$
\mathcal{L}=i \hat{\delta}_{+} \hat{\delta}_{-} \mathcal{F}
$$

In our case, the functional $\mathcal{F}$ is chosen as

$$
\mathcal{F}=B^{\mu} \partial_{\mu} \phi-i \chi^{\mu} \psi_{\mu}-i \chi \psi+V\left(\phi, B^{\rho}\right)
$$

where $V\left(\phi, B^{\rho}\right)$ is a potential. Therefore the Lagrangian $\mathcal{L}$ becomes

$$
\begin{aligned}
\mathcal{L} & =i \hat{\delta}_{+} \hat{\delta}_{-} \mathcal{F} \\
& =i \hat{\delta}_{+}\left\{\chi^{\mu} \partial_{\mu} \phi+B^{\mu} \partial_{\mu} \chi-i \chi^{\mu} H_{\mu}-i \chi H+\left(\chi \frac{\delta}{\delta \phi}\right) V\left(\phi, B^{\rho}\right)+\left(\chi^{\mu} \frac{\delta}{\delta B^{\mu}}\right) V\left(\phi, B^{\rho}\right)\right\} \\
& =i \hat{\delta}_{+}\left[\chi\left\{-\partial_{\mu} B^{\mu}+\frac{\delta}{\delta \phi} V\left(\phi, B^{\rho}\right)-i H\right\}+\chi^{\mu}\left\{\partial_{\mu} \phi+\frac{\delta}{\delta B^{\mu}} V\left(\phi, B^{\rho}\right)-i H_{\mu}\right\}\right] \\
& =\mathcal{L}_{B}+\mathcal{L}_{F}
\end{aligned}
$$

where $\mathcal{L}_{B}\left(\mathcal{L}_{F}\right)$ is the bosonic (fermionic) part of the Lagrangian,

$$
\begin{aligned}
\mathcal{L}_{B}= & i H\left\{-\partial_{\mu} B^{\mu}+\frac{\delta}{\delta \phi} V\left(\phi, B^{\rho}\right)-i H\right\}+i H^{\mu}\left\{\partial_{\mu} \phi+\frac{\delta}{\delta B^{\mu}} V\left(\phi, B^{\rho}\right)-i H_{\mu}\right\} \\
\mathcal{L}_{F}= & i \chi\left\{\partial_{\mu} \psi^{\mu}-\left(\psi \frac{\delta}{\delta \phi}\right) \frac{\delta}{\delta \phi} V\left(\phi, B^{\rho}\right)-\left(\psi^{\mu} \frac{\delta}{\delta B^{\mu}}\right) \frac{\delta}{\delta \phi} V\left(\phi, B^{\rho}\right)\right\} \\
& -i \chi^{\mu}\left\{\partial_{\mu} \psi+\left(\psi \frac{\delta}{\delta \phi}\right) \frac{\delta}{\delta B^{\mu}} V\left(\phi, B^{\rho}\right)+\left(\psi^{\nu} \frac{\delta}{\delta B^{\nu}}\right) \frac{\delta}{\delta B^{\mu}} V\left(\phi, B^{\rho}\right)\right\}
\end{aligned}
$$

Here we consider the only case that the potential is separated form as

$$
V\left(\phi, B^{\rho}\right)=V(\phi)+\frac{1}{2} B_{\mu} B^{\mu}
$$


As a result of taking this potential, $B^{\mu}$ has only zero solution in the large $\theta$ limit, and this is necessary condition to investigate the GMS soliton space. If there is non-trivial $B^{\mu}$ solution, then the moduli space is changed from GMS soliton moduli space and we are not interested in such a case. $\mathcal{L}_{B}$ and $\mathcal{L}_{F}$, therefore, become

$$
\begin{aligned}
& \mathcal{L}_{B}=i H\left\{-\partial_{\mu} B^{\mu}+\frac{\delta V(\phi)}{\delta \phi}-i H\right\}+i H^{\mu}\left(\partial_{\mu} \phi+B_{\mu}-i H_{\mu}\right), \\
& \mathcal{L}_{F}=i \chi\left\{\partial_{\mu} \psi^{\mu}-\psi \frac{\delta^{2} V(\phi)}{\delta \phi^{2}}\right\}-i \chi^{\mu}\left(\partial_{\mu} \psi+\psi_{\mu}\right) .
\end{aligned}
$$

Note that every product of fields should be defined by replacing the normal product by the Moyal product when we consider the noncommutative field theory.

\section{III.ii Commutative limit $\theta \rightarrow 0$}

We consider the balanced scalar model in 2-dimensional flat noncommutative space. One can get the theory on noncommutative space by changing the ordinary product into the star (Moyal) product $*_{\theta}$. Although noncommutativity is represented by the noncommutative parameter $\theta$ in the star product, this parameter is absorbed by the rescaling,

$$
\begin{aligned}
& x^{\mu} \rightarrow \sqrt{\theta} x^{\mu}, \\
& *_{\theta} \rightarrow *_{\theta=1} .
\end{aligned}
$$

However, the action is changed as follows:

$$
\begin{aligned}
& S_{B}=\int d^{2} x \theta\left[i H *\left\{-\frac{\partial_{\mu} B^{\mu}}{\sqrt{\theta}}+\frac{\delta V(* \phi)}{\delta \phi}-i H\right\}+i H^{\mu} *\left(\frac{\partial_{\mu} \phi}{\sqrt{\theta}}+B_{\mu}-i H_{\mu}\right)\right], \\
& S_{F}=\int d^{2} x \theta\left[i \chi *\left\{\frac{\partial_{\mu} \psi^{\mu}}{\sqrt{\theta}}-\psi \frac{\delta^{2} V(* \phi)}{\delta \phi^{2}}\right\}-i \chi^{\mu} *\left(\frac{\partial_{\mu} \psi}{\sqrt{\theta}}+\psi_{\mu}\right)\right],
\end{aligned}
$$

where $S_{B}\left(S_{F}\right)$ is the bosonic (fermionic) part of the action and $*$ means $*_{\theta=1}$ implicitly.

Let us consider the $\theta \rightarrow 0$ limit and calculate its partition function, which is compared with the $\theta \rightarrow \infty$ limit in the next subsection. The partition function is calculated as

$$
\mathcal{Z}=\int \mathcal{D} \phi \mathcal{D} \chi \mathcal{D} \psi \mathcal{D} \chi^{\mu} \mathcal{D} \psi^{\mu} \mathcal{D} B^{\mu} \mathcal{D} H \mathcal{D} H^{\mu} \exp \left(-S_{B}-S_{F}\right)
$$

The part of the action which contributes to the non-zero modes of $\phi$ and $H$ is

$$
\int d^{2} x \sqrt{\theta} i\left(H^{\mu} * \partial_{\mu} \phi-H * \partial_{\mu} B^{\mu}\right)
$$

We perform the integration of the non-zero modes of $H^{\mu}$ and $B^{\mu}$, and it yields delta functionals,

$$
\delta\left(\sqrt{2 \pi \theta} \partial_{\mu} \phi\right) \delta\left(\sqrt{2 \pi \theta} \partial_{\mu} H\right)
$$

Therefore, for $\phi$ and $H$, only zero modes integral remains. Next, the part of the action which contributes to the non-zero modes of $\chi$ and $\psi$ is

$$
\int d^{2} x \sqrt{\theta} i\left(\chi * \partial_{\mu} \psi^{\mu}-\chi^{\mu} * \partial_{\mu} \psi\right)
$$


Integration of non-zero modes of $\chi, \psi$ and $\chi^{\mu}, \psi^{\mu}$ yields the factor

$$
\left[\operatorname{det}\left(i \sqrt{\theta} \partial_{\mu}\right)\right]_{\chi_{\emptyset} \psi^{\mu}}\left[\operatorname{det}\left(i \sqrt{\theta} \partial_{\mu}\right)\right]_{\chi^{\mu} \psi_{\emptyset}}
$$

where the fields with the subscript $\emptyset$ denote non-zero modes. Therefore, for $\chi$ and $\psi$, only zero modes remain and the partition function $\mathcal{Z}$ becomes

$$
\begin{aligned}
\left.\left.\mathcal{Z}=\frac{\left[\operatorname{det}\left(i \sqrt{\theta} \partial_{\mu}\right)\right]_{\chi^{\mu} \psi_{\emptyset}}}{[\operatorname{det}(} \sqrt{2 \pi \theta} \partial_{\mu}\right)\right]_{H^{\mu} \phi_{\emptyset}} & \frac{\left[\operatorname{det}\left(i \sqrt{\theta} \partial_{\mu}\right)\right]_{\chi_{\emptyset} \psi^{\mu}}}{\left[\operatorname{det}\left(\sqrt{2 \pi \theta} \partial_{\mu}\right)\right]_{H_{\emptyset} B^{\mu}}} \\
& \times \int d y d \chi_{0} d \psi_{0} \mathcal{D} \chi^{\mu} \mathcal{D} \psi^{\mu} \mathcal{D} B^{\mu} d H_{0} \mathcal{D} H^{\mu} \exp \left(-S_{B}^{\prime}-S_{F}^{\prime}\right)
\end{aligned}
$$

where the fields with the subscript ${ }_{0}$ denote zero modes and the variable $y$ denotes the zero mode of $\phi$ (this is just a real constant number) and

$$
\begin{aligned}
& S_{B}^{\prime}=\int d^{2} x \theta\left[H_{0}\left\{H_{0}+i \frac{\delta V(y)}{\delta y}\right\}+H^{\mu} *\left(H_{\mu}+i B_{\mu}\right)\right], \\
& S_{F}^{\prime}=\int d^{2} x \theta i\left[-\chi_{0} \psi_{0} \frac{\delta^{2} V(y)}{\delta y^{2}}-\chi^{\mu} * \psi_{\mu}\right] .
\end{aligned}
$$

The factors in front of the integral in Eq.(III.20) cancel each other and only $[\operatorname{det}(2 \pi)]_{H^{\mu}}^{-1} \phi_{\emptyset}$ remains. $H^{\mu}, B^{\mu}$ and the zero mode of $H$ can be integrated out, and it yields the factor,

$$
\frac{1}{[v \theta / \pi]_{H_{0}}^{\frac{1}{2}}} \frac{1}{[\operatorname{det}(\theta / \pi)]_{H^{\mu} \phi_{0}}^{\frac{1}{2}}} \frac{1}{[\operatorname{det}(\theta / 4 \pi)]_{H_{0} B^{\mu}}^{\frac{1}{2}}}
$$

where $v$ is the volume of space-time. This volume is infinity but this will be canceled out by $\phi, \chi$ and $\psi$ zero modes integration, later. Integration of $\chi^{\mu}, \psi^{\mu}$ yields the factor $[\operatorname{det}(i \theta)]_{\chi^{\mu} \psi^{\mu}}$ , and the partition function $\mathcal{Z}$ becomes

$$
\mathcal{Z}=\sqrt{\frac{\pi}{v \theta}} \operatorname{det}(-1) \int d y d \chi_{0} d \psi_{0} \exp \left(-S_{B}^{\prime \prime}-S_{F}^{\prime \prime}\right),
$$

where

$$
\begin{aligned}
& S_{B}^{\prime \prime}=\int d^{2} x \frac{\theta}{4}\left\{\frac{\delta V(y)}{\delta y}\right\}^{2}, \\
& S_{F}^{\prime \prime}=-\int d^{2} x \theta i \chi_{0} \psi_{0} \frac{\delta^{2} V(y)}{\delta y^{2}} .
\end{aligned}
$$

Before integrating $y$, we expand $\delta V(y) / \delta y$ as

$$
\frac{\delta V(y)}{\delta y}=\left.y \frac{\delta^{2} V(y)}{\delta y^{2}}\right|_{y=y_{c}}+\mathcal{O}\left(y^{2}\right),
$$

where $y_{c}$ is a point of the extrema of the potential,

$$
\left.\frac{\delta V(y)}{\delta y}\right|_{y=y_{c}}=0
$$


and we should sum up all $y_{c}$ in the calculation of $\mathcal{Z}$ :

$$
\mathcal{Z}=\sqrt{\frac{\pi}{v \theta}} \operatorname{det}(-1) \sum_{y_{c}} \int d y d \chi_{0} d \psi_{0} \exp \left(-S^{\prime}\right),
$$

where

$$
S^{\prime}=v \theta\left[\frac{1}{4}\left\{\left.y \frac{\delta^{2} V(y)}{\delta y^{2}}\right|_{y=y_{c}}\right\}^{2}-\left.i \chi \psi \frac{\delta^{2} V(y)}{\delta y^{2}}\right|_{y=y_{c}}\right]
$$

Note that these zero modes do not depend on $x^{\mu}$, then the volume $v$ is factorized out. By integrating the zero modes of $\phi, \chi$ and $\psi$, we get the partition function,

$$
\begin{aligned}
\mathcal{Z} & =\sqrt{\frac{\pi}{v \theta}} \operatorname{det}(-1) \sum_{y_{c}} \frac{\left.i v \theta \frac{\delta^{2} V(y)}{\delta y^{2}}\right|_{y=y_{c}}}{\left\{\left.\frac{v \theta}{4 \pi} \frac{\delta^{2} V(y)}{\delta y^{2}}\right|_{y=y_{c}} ^{2}\right\}^{\frac{1}{2}}} \\
& =2 \pi i \operatorname{det}(-1) \sum_{y_{c}} \operatorname{sgn}\left[\left.\frac{\delta^{2} V(y)}{\delta y^{2}}\right|_{y=y_{c}}\right] .
\end{aligned}
$$

The factor in front of $\sum_{y_{c}}$ is removable as a normalizing factor.

Generally, the potential $V(\phi)$ is a polynomial of the scalar field $\phi$ :

$$
V(\phi)=b_{0}+b_{1} \phi+\frac{b_{2}}{2 !} \phi^{2}+\cdots+\frac{b_{m}}{m !} \phi^{m} \quad(m \geqslant 2)
$$

and so the result Eq. (III.31) becomes

$$
\mathcal{Z}=\left\{\begin{array}{cc}
\operatorname{sgn}\left[b_{m}\right] & : m \text { is even number } \\
0 & : m \text { is odd number. }
\end{array}\right.
$$

In the limit $\theta \rightarrow 0$, it seems that the kinetic term plays a dominant role, but the effect of the potential term survives. By this effect, degree of the polynomial completely determine the partition function as cyclic form of Eq. (III.33).

\section{III.iii Noncommutative limit $\theta \rightarrow \infty$}

In the strong noncommutative limit $\theta \rightarrow \infty$, the terms that have derivatives are effectively ignored, and the remaining terms which are potential and mass terms in the balanced scalar model determine the field configuration. In the noncommutative space, there is a specific field configuration, that is GMS soliton 14. This soliton is the solution of the field equation, and there are infinite solutions. Hence, the partition function is sum of contributions from infinite vacuum states.

In the large $\theta$ limit, the balanced scalar model Eqs.([II.10, [II.11) is written as

$$
S=S_{B}+S_{F}
$$


where

$$
\begin{aligned}
& S_{B}=\int d^{2} x \theta\left[H *\left\{\frac{\delta V(* \phi)}{\delta \phi}-H\right\}+H^{\mu} *\left(B_{\mu}-H_{\mu}\right)\right], \\
& S_{F}=\int d^{2} x \theta\left[-i \chi *\left(\psi \frac{\delta}{\delta \phi}\right) \frac{\delta V(* \phi)}{\delta \phi}+i \chi^{\mu} * \psi_{\mu}\right] .
\end{aligned}
$$

After integrating over the fields $H, H^{\mu}$, the action is

$$
S=\int d^{2} x \theta\left[\frac{1}{4} \frac{\delta V(* \phi)}{\delta \phi} \frac{\delta V(* \phi)}{\delta \phi}-i \chi *\left(\psi \frac{\delta}{\delta \phi}\right) \frac{\delta V(* \phi)}{\delta \phi}+\frac{1}{4} B^{\mu} * B_{\mu}+i \chi^{\mu} * \psi_{\mu}\right]
$$

Integration of the fields $B^{\mu}, \chi^{\mu}$ and $\psi_{\mu}$ is simply performed, and the partition function is written as

$$
\mathcal{Z}=\int \mathcal{D} \phi \mathcal{D} \chi \mathcal{D} \psi e^{-S}
$$

where the action is

$$
S=\int d^{2} x \theta\left[\frac{1}{4} \frac{\delta V(* \phi)}{\delta \phi} \frac{\delta V(* \phi)}{\delta \phi}-i \chi *\left(\psi \frac{\delta}{\delta \phi}\right) \frac{\delta V(* \phi)}{\delta \phi}\right] .
$$

In the large $\theta$ limit, the derivative terms are irrelevant, and the potential terms dominates ${ }^{a}$. The field configuration is determined by the form of the potential. In particular the stationary field configuration is obtained by solving the field equation:

$$
\frac{\delta V(* \phi)}{\delta \phi}=0
$$

In the calculation of the partition function, we should treat these GMS soliton as the stationary points, and the quantum fluctuation is a perturbation from the GMS soliton. In the following section, we discuss the treatment of the GMS soliton in the partition function, and understand the GMS soliton from a topological view point.

We consider the $\phi^{m+2}$ potential,

$$
V(\phi)=\{\phi \text {-polynomial of }(m+2) \text { degree }\},
$$

and the field equation takes the following factorized form,

$$
\frac{\delta V}{\delta \phi}=b_{m} \phi *\left(\phi-v_{1}\right) *\left(\phi-v_{2}\right) * \cdots *\left(\phi-v_{m}\right)
$$

Where we assume the $v_{i}$ are real constant numbers with $v_{1}<v_{2}<\cdots<v_{m-1}<v_{m}$. The GMS soliton is given by

$$
\phi_{G M S}=\lambda_{i} \mathbf{P}_{i}
$$

\footnotetext{
${ }^{a)}$ Actually kinetic term integral is survived for next order integral but it yield nothing because of the BRST symmetry.
} 


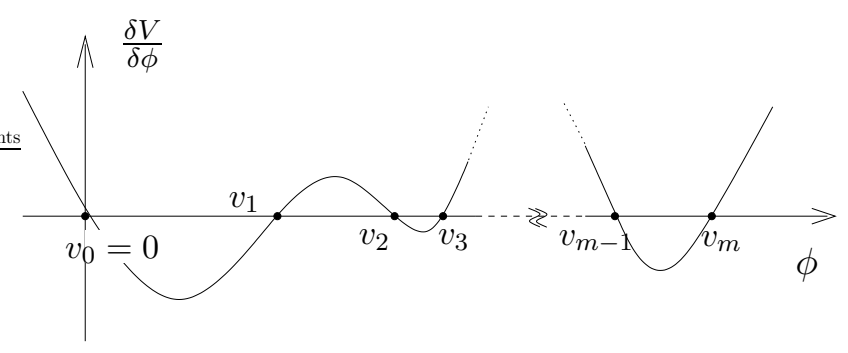

Figure 2: $\frac{\delta V}{\delta \phi}, b_{m}>0$ and $m$ is odd.

where $\mathbf{P}_{i}$ is a projection, and satisfies the idempotent relation $\mathbf{P}_{i} * \mathbf{P}_{j}=\delta_{i j} \mathbf{P}_{i}$. The coefficient $\lambda_{i}$ is determined by Eq.(III.9). In the Moyal plane, this projection is given by the Laguerre polynomial $2(-1)^{i} e^{-x^{2}} \mathrm{~L}_{i}\left(2 x^{2}\right)$ 14). It is possible to chose concrete representation of corresponding Weyl mapped projection operator, for example

$$
\hat{\mathbf{P}}_{i}=|i\rangle\langle i|,
$$

where $|i\rangle$ is the number representation base 17, 17). The Weyl mapped GMS soliton is written as

$$
\hat{\phi}_{G M S}=\lambda_{i} \hat{\mathbf{P}}_{i}
$$

We substitute the GMS solution to $\phi$ of Eq.(III.9)

$$
\left.\frac{\delta V(\hat{\phi})}{\delta \hat{\phi}}\right|_{G M S}=b_{m} \lambda_{i}\left(\lambda_{i}-v_{1}\right)\left(\lambda_{i}-v_{2}\right) \cdots\left(\lambda_{i}-v_{m}\right) \mathbf{P}_{i}=0 .
$$

The solutions of Eq.(III.13) are given by

$$
\lambda_{i}=0, v_{1}, v_{2}, \cdots, v_{m}
$$

These solutions are coefficients of the GMS soliton. The general GMS solution is a linear combination of the projections,

$$
\begin{aligned}
\hat{\phi}_{G M S} & =\sum_{i} \lambda_{i} \hat{\mathbf{P}}_{i} \\
& =v_{1} \sum_{i \in \mathbf{S}_{1}} \hat{\mathbf{P}}_{i}+v_{2} \sum_{i \in \mathbf{S}_{2}} \hat{\mathbf{P}}_{i}+\cdots+v_{m} \sum_{i \in \mathbf{S}_{m}} \hat{\mathbf{P}}_{i} \\
& =v_{1} \hat{\mathbf{P}}_{\mathbf{S}_{1}}+v_{2} \hat{\mathbf{P}}_{\mathbf{S}_{2}}+\cdots+v_{m} \hat{\mathbf{P}}_{\mathbf{S}_{m}},
\end{aligned}
$$

where $\mathbf{S}_{A}(A \in\{1,2, \cdots, m\})$ is a set of the indices of the projections, and it is defined as if the coefficient of a projection $\hat{\mathbf{P}}_{i}$ is $v_{A}$ in (III.15) then the index " $i$ " belongs to $\mathbf{S}_{A}$ - For example, if a GMS soliton takes the form as $\hat{\phi}_{G M S}=v_{1}\left(\hat{\mathbf{P}}_{1}+\hat{\mathbf{P}}_{2}\right)+v_{2}\left(\hat{\mathbf{P}}_{0}\right)$, then $\mathbf{S}_{1}=\{1,2\}$ and $\mathbf{S}_{2}=\{0\}$. We define $\hat{\mathbf{P}}_{\mathbf{S}_{A}} \equiv \sum_{i \in \hat{\mathbf{S}}_{A}} \mathbf{P}_{i}$. For $A \neq B(A, B \in\{1,2, \cdots, m\})$ the sets $\mathbf{S}_{A}$ and $\mathbf{S}_{B}$ are disjoint each other $\mathbf{S}_{A} \cap \mathbf{S}_{B}=\varnothing$, and the projections are orthogonal, $\hat{\mathbf{P}}_{\mathbf{S}_{A}} \cdot \hat{\mathbf{P}}_{\mathbf{S}_{B}}=0$. In the commutative space, the field equation Eq.(III.9) has only constant 
solutions. On the contrary, in the noncommutative space, there exist GMS solitons that are operators for Hilbert space of quantized coordinate, and this fact make new solutions with linear combination of projections. The indices of the projection are not bounded above, then the number of the element of $\mathbf{S}_{A}\left(\operatorname{rank} \mathbf{S}_{A}\right)$ is allow to be infinite. In the following, we introduce a cut-off $N$ of total number of the $\operatorname{rank} \mathbf{S}_{A}$, and then there are $N$ projections $\left\{\hat{\mathbf{P}}_{0}, \hat{\mathbf{P}}_{1}, \hat{\mathbf{P}}_{2}, \cdots, \hat{\mathbf{P}}_{N-1}\right\}$. After the calculation we take the limit $N \rightarrow \infty$.

Next step, we perform Gaussian integral around each vacuum. Note that, in the commutative space the number of the solution is $(m+1)$, on the contrary in the noncommutative space there are $(m+1)^{N}$ solutions. In the commutative space, we can take the quantum fluctuation from the vacua of the finite number of simply constant solutions of Eq.(III.9). On the contrary, there are infinite GMS solitons in noncommutative space. Let us take the quantum fluctuation around each of the GMS solitons as $\phi=\left.\phi\right|_{G M S}+\phi_{q}$. We expand the Lagrangian around the specific GMS soliton,

$$
\mathcal{L}_{\mathrm{S}}=\left.\left.\frac{1}{4}\left(\phi_{q} \frac{\delta}{\delta \phi}\right) \frac{\delta V(* \phi)}{\delta \phi}\right|_{G M S} *\left(\phi_{q} \frac{\delta}{\delta \phi}\right) \frac{\delta V(* \phi)}{\delta \phi}\right|_{G M S}-\left.i \chi *\left(\psi \frac{\delta}{\delta \phi}\right) \frac{\delta V(* \phi)}{\delta \phi}\right|_{G M S}
$$

The subscript $\mathbf{S}$ of $\mathcal{L}$ means that the Lagrangian is expanded around the GMS soliton specified by $\mathbf{S}$ that is the family of $\left\{\mathbf{S}_{1}, \mathbf{S}_{2}, \cdots, \mathbf{S}_{m}\right\}$. . On the Moyal plane, the field operators $\hat{\phi}_{q}, \hat{\chi}$ and $\hat{\psi}$ are expanded as

$$
\begin{aligned}
\hat{\phi}_{q} & =\sum_{i, j=0}^{\infty} \phi_{i j}|i\rangle\langle j|, \\
\hat{\chi} & =\sum_{i, j=0}^{\infty} \chi_{i j}|i\rangle\langle j|, \\
\hat{\psi} & =\sum_{i, j=0}^{\infty} \psi_{i j}|i\rangle\langle j| .
\end{aligned}
$$

The field operators $\hat{\phi}_{q}, \hat{\chi}$ and $\hat{\psi}$ are Hermitian operators,

$$
\phi_{q}^{\dagger}=\phi_{q}, \chi^{\dagger}=\chi, \psi^{\dagger}=\psi .
$$

We rewrite the field such as $\phi_{i j}=\phi_{i j}^{R}+i \phi_{i j}^{I}\left(\phi_{i j}^{R}, \phi_{i j}^{I} \in \mathbf{R}\right)$. Then the hermitian conditions need that real (imaginary) parts are symmetric (anti-symmetric)

$$
\begin{cases}\phi_{i j}^{R}=\phi_{j i}^{R}, & \phi_{i j}^{I}=-\phi_{j i}^{I}, \\ \chi_{i j}^{R}=\chi_{j i}^{R}, & \chi_{i j}^{I}=-\chi_{j i}^{I}, \\ \psi_{i j}^{R}=\psi_{j i}^{R}, & \psi_{i j}^{I}=-\psi_{j i}^{I} .\end{cases}
$$

In the operator picture, the integration in the action is replaced by the trace over the field operators. The lagrangian is always described as the Weyl mapped lagrangian

$$
S_{\mathbf{S}}=\operatorname{Tr} \hat{\mathcal{L}}_{\mathbf{S}}=S_{B}^{\mathbf{S}}+S_{F}^{\mathbf{S}},
$$

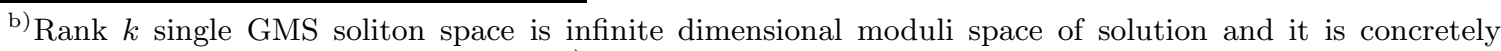
described as the coset space $\frac{U(N)}{U(k) U(N-k)}$ 13). In our case, GMS solution space contains not only single soliton but also multi-solitons that have any rank solutions. The moduli space specified by $\mathbf{S}$ is $\frac{U(N)}{U\left(n_{0}\right) U\left(n_{1}\right) \cdots U(N-1)}$, where $n_{i}$ is the rank of $\mathbf{S}_{i}$.
} 
where $S_{B}^{\mathbf{S}}$ and $S_{F}^{\mathbf{S}}$ are given by

$$
\begin{aligned}
S_{B}^{\mathbf{S}} & =\left.\left.\frac{1}{4} \theta \operatorname{Tr}\left(\hat{\phi}_{q} \frac{\delta}{\delta \hat{\phi}}\right) \frac{\delta V(\hat{\phi})}{\delta \hat{\phi}}\right|_{G M S}\left(\hat{\phi}_{q} \frac{\delta}{\delta \hat{\phi}}\right) \frac{\delta V(\hat{\phi})}{\delta \hat{\phi}}\right|_{G M S}, \\
S_{F}^{\mathbf{S}} & =-\left.i \theta \operatorname{Tr} \hat{\chi}\left(\hat{\psi} \frac{\delta}{\delta \hat{\phi}}\right) \frac{\delta V(\hat{\phi})}{\delta \hat{\phi}}\right|_{G M S} .
\end{aligned}
$$

Firstly, we consider the bosonic part of the action. The second derivative of the potential is

$$
\begin{aligned}
\left(\hat{\phi}_{q} \frac{\delta}{\delta \hat{\phi}}\right) & \left.\frac{\delta V(\hat{\phi})}{\delta \hat{\phi}}\right|_{G M S} \\
= & \left.\left(\hat{\phi}_{q} \frac{\delta}{\delta \hat{\phi}}\right)\left\{b_{m} \hat{\phi}\left(\hat{\phi}-v_{1}\right)\left(\hat{\phi}-v_{2}\right) \cdots\left(\hat{\phi}-v_{m}\right)\right\}\right|_{G M S} \\
= & b_{m}\left[\hat{\phi}_{q}\left(\hat{\phi}-v_{1}\right)\left(\hat{\phi}-v_{2}\right) \cdots\left(\hat{\phi}-v_{m}\right)\right. \\
& +\hat{\phi} \hat{\phi}_{q}\left(\hat{\phi}-v_{2}\right) \cdots\left(\hat{\phi}-v_{m}\right) \\
& +\hat{\phi}\left(\hat{\phi}-v_{1}\right) \phi_{q} \cdots\left(\hat{\phi}-v_{m}\right) \\
& \vdots \\
& \left.+\hat{\phi}\left(\hat{\phi}-v_{1}\right)\left(\hat{\phi}-v_{2}\right) \cdots \hat{\phi}_{q}\right]_{\hat{\phi}=\hat{\phi}_{G M S} .}
\end{aligned}
$$

We put the GMS soliton into Eq.([II.23), and we get

$$
\begin{aligned}
\left(\hat{\phi}_{q} \frac{\delta}{\delta \hat{\phi}}\right) & \left.\frac{\delta V(\hat{\phi})}{\delta \hat{\phi}}\right|_{G M S} \\
= & b_{m} \sum_{A=0}^{m} \sum_{B=A}^{m} \prod_{i=0}^{A-1}\left(v_{B}-v_{i}\right) \sum_{C=0}^{A} \prod_{j=A+1}^{m}\left(v_{C}-v_{j}\right) \hat{\mathbf{P}}_{\mathbf{S}_{B}} \hat{\phi} \hat{\mathbf{P}}_{\mathbf{S}_{C}} \\
= & b_{m} \sum_{A=0}^{m} \prod_{i=0}^{A-1}\left(v_{A}-v_{i}\right) \prod_{j=A+1}^{m}\left(v_{A}-v_{j}\right) \hat{\mathbf{P}}_{\mathbf{S}_{A}} \hat{\phi}_{q} \hat{\mathbf{P}}_{\mathbf{S}_{A}} \\
& +b_{m} \sum_{\substack{A>B \\
A, B \in\{0, \cdots, r\}}} \sum_{k=B}^{A} \prod_{i=0}^{k-1}\left(v_{A}-v_{i}\right) \prod_{j=k+1}^{m}\left(v_{B}-v_{j}\right) \hat{\mathbf{P}}_{\mathbf{S}_{A}} \hat{\phi}_{q} \hat{\mathbf{P}}_{\mathbf{S}_{B}},
\end{aligned}
$$

where we define the zero ${ }^{\text {th }}$ solution and the zero $^{\text {th }}$ projection by

$$
v_{0} \equiv 0, \quad \hat{\mathbf{P}}_{\mathbf{S}_{0}} \equiv \hat{\mathbf{1}}-\hat{\mathbf{P}}_{\mathbf{S}_{1}}-\hat{\mathbf{P}}_{\mathbf{S}_{2}}-\cdots-\hat{\mathbf{P}}_{\mathbf{S}_{r}}
$$

and we introduce the following symbols for convenience

$$
\prod_{i=0}^{-1}\left(v_{0}-v_{i}\right) \equiv 1, \quad \prod_{j=m+1}^{m}\left(v_{m}-v_{j}\right) \equiv 1 .
$$


The coefficient of the cross term $\hat{\mathbf{P}}_{\mathbf{S}_{A}} \hat{\phi}_{q} \mathbf{P}_{\mathbf{S}_{B}}(A>B)$ in Eq.(III.24) is

$$
\sum_{k=B}^{A} \prod_{i=0}^{k-1}\left(v_{A}-v_{i}\right) \prod_{j=k+1}^{m}\left(v_{B}-v_{j}\right)
$$

Eq. [II.27) is written as

$$
\begin{aligned}
\sum_{k=B}^{A} \prod_{i=0}^{k-1}\left(v_{A}-v_{i}\right) & \prod_{j=k+1}^{m}\left(v_{B}-v_{j}\right) \\
= & \quad \begin{aligned}
&\left(v_{A}-v_{0}\right)\left(v_{A}-v_{1}\right) \cdots\left(v_{A}-v_{B-1}\right) \\
& \quad \quad \times\left(v_{A+1}-v_{B}\right)\left(v_{A+2}-v_{B}\right) \cdots\left(v_{m-1}-v_{B}\right)\left(v_{m}-v_{B}\right) \$_{A-B}
\end{aligned}
\end{aligned}
$$

where $\$_{A-B}$ is defined as

$$
\begin{aligned}
\$_{A-B} \equiv & \left(v_{B}-v_{B+1}\right)\left(v_{B}-v_{B+2}\right) \cdots\left(v_{B}-v_{A}\right) \\
& +\left(v_{A}-v_{B}\right)\left(v_{B}-v_{B+2}\right) \cdots\left(v_{B}-v_{A}\right) \\
& \vdots \\
& +\left(v_{A}-v_{B}\right)\left(v_{B+1}-v_{A}\right) \cdots\left(v_{A-1}-v_{A}\right) .
\end{aligned}
$$

We can see $\$_{A-B}$ always vanishes for any set of $v_{i}$ (see Appendix $\mathrm{D}$ ). Then the cross term does not appear. Finally the remaining terms are closed in each set $\mathbf{S}_{A}$ such as

$$
\left.\left(\hat{\phi}_{q} \frac{\delta}{\delta \hat{\phi}}\right) \frac{\delta V(\hat{\phi})}{\delta \hat{\phi}}\right|_{G M S}=b_{m} \sum_{A=0}^{m} \prod_{i=0}^{A-1}\left(v_{A}-v_{i}\right) \prod_{j=A+1}^{m}\left(v_{A}-v_{j}\right) \hat{\mathbf{P}}_{\mathbf{S}_{A}} \hat{\phi}_{q} \hat{\mathbf{P}}_{\mathbf{S}_{A}}
$$

Then the bosonic part of the action is written as

$$
S_{B}^{\mathbf{S}}=\frac{1}{4} \theta \sum_{A=0}^{m}\left(b_{m} \prod_{i=0}^{A-1}\left(v_{A}-v_{i}\right) \prod_{j=A+1}^{m}\left(v_{A}-v_{j}\right)\right)^{2} \operatorname{Tr}\left[\hat{\mathbf{P}}_{\mathbf{S}_{A}} \hat{\phi}_{q} \hat{\mathbf{P}}_{\mathbf{S}_{A}} \hat{\phi}_{q}\right]
$$

For the fermionic part, the calculation is the same as the bosonic part. $\left(\hat{\psi} \frac{\delta}{\delta \hat{\phi}}\right) \frac{\delta V(\hat{\phi})}{\delta \hat{\phi}}$ is given as

$$
\left.\left(\hat{\psi} \frac{\delta}{\delta \hat{\phi}}\right) \frac{\delta V(\hat{\phi})}{\delta \hat{\phi}}\right|_{G M S}=b_{m} \sum_{A=0}^{m} \prod_{i=0}^{A-1}\left(v_{A}-v_{i}\right) \prod_{j=A+1}^{m}\left(v_{A}-v_{j}\right) \hat{\mathbf{P}}_{\mathbf{S}_{A}} \hat{\psi} \hat{\mathbf{P}}_{\mathbf{S}_{A}}
$$

Then the fermionic part of the action is written as

$$
S_{F}^{\mathbf{S}}=-i \theta b_{m} \sum_{A=0}^{m} \prod_{i=0}^{A-1}\left(v_{A}-v_{i}\right) \prod_{j=A+1}^{m}\left(v_{A}-v_{j}\right) \operatorname{Tr}\left[\hat{\chi} \hat{\mathbf{P}}_{\mathbf{S}_{A}} \hat{\psi} \hat{\mathbf{P}}_{\mathbf{S}_{A}}\right] .
$$


We substitute $\mathbf{P}_{\mathbf{S}_{A}}=\sum_{i \in \mathbf{S}_{A}} \mathbf{P}_{i}$ into Eqs.(III.31, III.33), and use Eq.(III.17), then we get the action,

$$
\begin{aligned}
S_{\mathbf{S}} & =S_{B}^{\mathbf{S}}+S_{F}^{\mathbf{S}} \\
& =\sum_{A=0}^{m}\left\{\frac{1}{4} \theta \sum_{i, j \in \mathbf{S}_{A}}\left(b_{m} \zeta_{A}\right)^{2} \phi_{i j} \phi_{j i}-i \theta \sum_{i, j \in \mathbf{S}_{A}} b_{m} \zeta_{A} \chi_{i j} \psi_{j i}\right\},
\end{aligned}
$$

where we define

$$
\zeta_{A} \equiv \prod_{k=0}^{A-1}\left(v_{A}-v_{k}\right) \prod_{l=A+1}^{m}\left(v_{A}-v_{l}\right), \quad A \in\{0,1,2, \cdots, m\} .
$$

Note that the $\phi_{i j}, \chi_{i j}$ and $\psi_{i j}$ are the c-numbers. Using the result in Appendix C, we can estimate both the partition function in the operator representation and in the commutative field representation with the Moyal product, but the results have no difference. Here, we perform the integral in the operator representation. Then the path-integral becomes simply an integral of real number (real Grassmann number) .

$$
\begin{aligned}
\mathcal{Z}_{\mathbf{S}} & =\int \mathcal{D} \chi \mathcal{D} \psi \mathcal{D} \phi e^{-S_{\mathbf{S}}} \\
& =\int \prod_{A=0}^{m} \prod_{\substack{m>n \\
i, j \in \mathbf{S}_{A}}}\left(\frac{d \phi_{i j}}{\sqrt{4 \pi \theta}}\right) d \chi_{i j} d \psi_{i j} \exp \left\{-S_{B}^{\mathbf{S}}-S_{F}^{\mathbf{S}}\right\} \\
& =\prod_{A=0}^{m}\left[\sqrt{b_{m} \zeta_{A}}\right]^{-n_{A}^{2}}\left[b_{m} \zeta_{A}\right]^{n_{A}^{2}}
\end{aligned}
$$

and we get

$$
\mathcal{Z}_{\mathbf{S}}=\prod_{A=0}^{m}\left[\operatorname{sgn}\left(b_{m} \zeta_{A}\right)\right]^{n_{A}}
$$

Here $n_{A}$ is the number of the elements belong to the set $\mathbf{S}_{A}$, and we call this number the "rank" of $\mathbf{S}_{A} ; n_{A}=\operatorname{rank} \mathbf{S}_{A}$. The noncommutative parameter $\theta$ contained under integral measure $d \phi$ in the second line of (III.36) comes from the integral of $H$. The path integral of the fields $\phi_{i j}, \chi_{i j}$ and $\psi_{i j}$ with indices $\left(i \in \mathbf{S}_{A}, j \in \mathbf{S}_{B}, A \neq B\right)$ is done with the weight of the kinetic terms, which contributes "1" in the partition function. The number of the GMS solitons specified by $\mathbf{S}=\left\{\mathbf{S}_{\mathbf{1}}, \mathbf{S}_{\mathbf{2}}, \cdots, \mathbf{S}_{\mathbf{m}}\right\}$ is $\frac{N !}{n_{0} ! n_{1} ! \cdots n_{m} !}$, where $n_{i}=\operatorname{rank} \mathbf{S}_{\mathbf{i}}$. Therefore the total partition function that includes all the GMS solitons is given by

$$
\begin{aligned}
\mathcal{Z}_{\text {Total }} & =\lim _{N \rightarrow \infty} \sum_{\mathbf{S}} \mathcal{Z}_{\mathbf{S}} \\
& =\lim _{N \rightarrow \infty} \sum_{\substack{n_{0}, \cdots, n_{m}=0 \\
n_{0}+\cdots+n_{m}=N}}^{N} \frac{N !}{n_{0} ! n_{1} ! \cdots n_{m} !} \prod_{A=0}^{m}\left[\operatorname{sgn}\left(b_{m} \zeta_{A}\right)\right]^{n_{A}} \\
& =\lim _{N \rightarrow \infty}\left(\operatorname{sgn}\left[b_{m}\right]\right)^{N}\left(\operatorname{sgn} \zeta_{0}+\operatorname{sgn} \zeta_{1}+\cdots+\operatorname{sgn} \zeta_{m}\right)^{N} .
\end{aligned}
$$


Under the same condition of $v_{0}<v_{1}<\cdots<v_{m}$ as previous section (see fig.2 too), the sign of $\zeta_{A}$ is simple form,

$$
\operatorname{sgn}\left(\zeta_{A}\right)=(-1)^{m-A}, \quad A \in\{0,1,2, \cdots, m\} .
$$

Then the partition function is

$$
\begin{aligned}
\mathcal{Z}_{\text {Total }} & =\lim _{N \rightarrow \infty}\left(\operatorname{sgn}\left[b_{m}\right]\right)^{N}(-1)^{m}(\underbrace{1-1+1-1+\cdots}_{m+1})^{N} \\
& = \begin{cases}\lim _{N \rightarrow \infty}\left(\operatorname{sgn}\left[b_{m}\right]\right)^{N} & : m \text { is even number } \\
0 & : m \text { is odd number. }\end{cases}
\end{aligned}
$$

Note that through a simple calculation, the partition function Eq.(III.38) is rewritten as,

$$
\begin{aligned}
\mathcal{Z}_{\text {Total }}=\lim _{N \rightarrow \infty}\left[\operatorname{sgn}\left(b_{m}\right)\right]^{N}(-1)^{m} \\
\quad \times \sum_{n_{p}=0}^{N}(-1)^{n_{p}} \frac{N !}{\left(N-n_{p}\right) ! n_{p} !}[(m+1) / 2]^{n_{p}}([m / 2]+1)^{N-n_{p}},
\end{aligned}
$$

where the bracket $[\cdots]$ is the Gauss's notation. The integer $n_{p}$ is the number of the negative solutions, and it is the sum of $n_{2 i+1}$. This number $n_{p}$ is related to the Morse theory. This is discussed in the next section.

\section{Euler number of GMS soliton space}

In this section we discuss the properties of the partition function in the point of view of quantum geometry.

\section{IV.i Noncommutative Mathai-Quillen formalism}

In the beginning, we study the partition function with Mathai-Quillen formalism.

As in the usual cohomological field theory, $\psi$ is a tangent vector to the zero-section, i.e. the tangent vector to the solution space, $\left\{\phi, B_{\mu} \mid s^{a}\left(\phi, B_{\mu}, \theta\right)=0\right\}=\mathcal{M}$. Note that the vanishing theorem asserts that the zero-section space is identified with the GMS solution space. In our theory, since $B^{\mu} B_{\mu}=0$ has only a trivial solution, the zero section space is identified with $\left\{\phi \mid \frac{\delta V}{\delta \phi}=0\right\}$. A condition like the vanishing theorem appears in general in the balanced topological field theory.

In the commutative limit $(\theta \rightarrow 0)$, the relevant terms of the bosonic action are

$$
\left|\partial_{\mu} \phi\right|^{2}+\left|\frac{\delta V}{\delta \phi}\right|^{2} .
$$

So the lowest energy solutions (true vacua) are constant that satisfy $\frac{\delta V}{\delta \phi}=0$. The number

of the solutions is $m+1$, that is the exponent of $\frac{\delta V}{\delta \phi}$ in the commutative limit. Hence, there 
is no problem in defining the Euler number of the space $\mathcal{M}$ which consists of $m+1$ isolate points as $\pm \sum_{k=0}^{m}(-1)^{k}$. Contrary, in the large $\theta$ limit, the number of GMS soliton solutions is infinite, that is rank of $K_{0}$. Generally, this is difficult case to define the Euler number of $\mathcal{M}$. However, as we saw in the previous sections, the partition function is invariant under the shift of $\theta$. Finally, the Euler number of the moduli space is invariant under the noncommutative deformation of $\frac{\delta V}{\delta \phi}=0$.

Gaussian integral in the noncommutative field theories is not familiar in general. But in this case, it is well-defined by the supersymmetry. As we see at first, the result of the path integral in the strong noncommutative limit does not contradict to the commutative limit. This is an evidence of validity of the Gaussian integral of the noncommutative field theory. Note that the large $\theta$ limit is not the strong coupling limit. We have to consider two parameters, the noncommutative parameter $\theta$ and the coupling constant $g$ multiplying overall. In order to get exact results by perturbative calculations, it is necessary for $g$ to approach to zero.

In the cohomological field theory, the partition function does not depend on an overall parameter like this $g$.

We summarize the previous sections by taking account of the fact that the partition function of cohomological field theory can be regard as the Euler number of $\mathcal{M}$ by MathaiQuillen formalism 4 .

Theorem IV.1 ( $\theta$-shift invariants) The partition functions $Z_{\theta}$ of the noncommutative cohomological field theories are invariant under the shift of the noncommutative parameter $\theta$.

$$
\frac{\delta}{\delta \theta} Z_{\theta}=0
$$

Theorem IV.2 (Euler number of GMS solution space) On the Moyal plane, when the GMS solution space for real scalar field $\phi$ is

$$
\mathcal{M}_{m}=\left\{\phi \mid b_{m}\left(\cdots(\underbrace{\left.\left.\phi *_{\theta}\left(\phi-v_{1}\right)\right) *_{\theta}\left(\phi-v_{2}\right)\right) \cdots *_{\theta}\left(\phi-v_{m}\right)}_{m+1}=0, b_{m}>0\}\right.\right.
$$

and any two of $v_{i}$ are different $\left(v_{i} \neq v_{j}\right.$ for $\left.i \neq j\right)$, then the Euler number of the space $\mathcal{M}_{m}$ is given by

$$
\chi_{m}= \begin{cases}1 & : m \text { is even number } \\ 0 & : m \text { is odd number. }\end{cases}
$$

\section{IV.ii Noncommutative Morse Theory}

In this subsection, we see that the noncommutative cohomological field theory can be regarded as the noncommutative Morse theory 19, 24].

In the commutative limit, the partition function of our theory is given by the Hessian

of the $\frac{\delta^{2} V}{\delta \phi^{2}}$ as we saw in the section III. Especially, the zero mode of $\partial_{\mu} \phi=0$ is just a real 
constant number, then the determinant of the Hessian at the critical point $p$ is determined by the sign of the second derivative of the real function at $p$, where the critical point $p$ is the solution of $\left.\frac{d V(x)}{d x}\right|_{p}=0 \quad(x \in \mathbf{R})$. We denote the number of negative eigenvalues of the Hessian as $n_{p}$. In the commutative limit $n_{p}$ is either 0 or 1 , so the partition function is written by

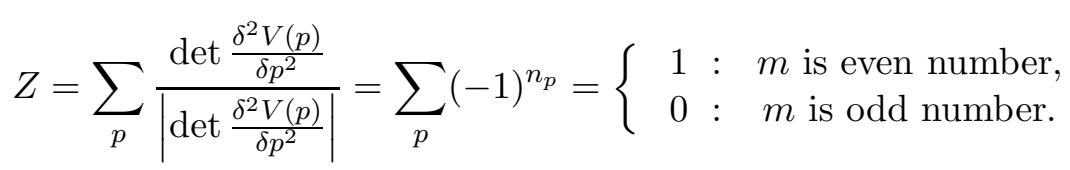

By the fundamental theorem of Morse theory, this is the Euler number of the isolated points $\{p\}$ and this result is consistent with the result of applying Mathai-Quillen formalism to cohomological field theory.

On the other hand, the partition function is determined by the determinant of the operator valued Hessian $\frac{\delta^{2} V(\phi)}{\delta \phi^{2}}$ in the large $\theta$ limit. The critical points are operators too, i.e.GMS solitons are critical points and they exist infinitely at each $p$. But for each critical point, we can estimate $\operatorname{det}\left(\frac{\delta^{2} V(\phi)}{\delta \phi^{2}}\right)$. Now we introduce the Morse index $M_{n_{p}} . M_{n_{p}}$ is defined as the number of the GMS solitons satisfying the condition that Hessian consisting of the GMS solitons has $n_{p}$ negative eigenvalues. As we saw in the section III and the Appendix, the $n_{p}$ is the number of projection operators $\mathbf{P}_{i}$ whose coefficient belong to $p_{-}$in the GMS solution. Here we define $p_{-}$and $p_{+}$as the set of the critical points that satisfy

$$
\begin{aligned}
& \left.\frac{\delta^{2} V(p)}{\delta p^{2}}\right|_{p_{-}}<0 \\
& \left.\frac{\delta^{2} V(p)}{\delta p^{2}}\right|_{p_{+}}>0
\end{aligned}
$$

In the case of positive $b_{m}$, the critical points are, $p_{-}=\left\{v_{0}, v_{2}, \cdots, v_{m}\right\}$, and $p_{+}=$ $\left\{v_{1}, v_{3}, \cdots, v_{m-1}\right\}$ (see Fig.2). The number of the $p_{-}$elements is $[(m+1) / 2]$, and the number of the $p_{+}$is $[m / 2]+1$. Then the number of combinations that $n_{p}$ projections is combined with $[(m+1) / 2]$ points of $p_{-}$in the soliton is $[(m+1) / 2]^{n_{p}}$. When the total number of projections is fixed by $N$, then the remaining $\left(N-n_{p}\right)$ projections are combined with $p_{+}$ and its number of combinations is $([m / 2]+1)^{\left(N-n_{p}\right)}$. Then the Morse index is given by

$$
M_{n_{p}}(m, N)=[(m+1) / 2]^{n_{p}}([m / 2]+1)^{\left(N-n_{p}\right)} \cdot \frac{N !}{\left(N-n_{P}\right) ! n_{p} !} .
$$

$M_{n_{p}}$ is divergent in the limit of $N \rightarrow \infty$. But we can define the Euler number of the isolate GMS soliton solutions as the fundamental theorem of Morse theory,

$$
\begin{aligned}
\chi_{m} & =\sum_{G M S}(-1)^{n_{p}}=\sum_{n_{p}}(-1)^{n_{p}} M_{n_{p}}=(-[(m+1) / 2]+[m / 2]+1)^{N} \\
& =\left\{\begin{aligned}
1: & m \text { is even number } \\
0: & m \text { is odd number. }
\end{aligned}\right.
\end{aligned}
$$

This is a consistent result with the section III and the Mathai-Quillen formalism. Therefore we can conclude that the noncommutative cohomological field theory makes it possible to generalize the Morse theory for the noncommutative field theory. 


\section{Conclusion and Discussion}

We have studied the noncommutative cohomological field theory. Especially, the balanced scalar model is investigated carefully. A couple of theorems is provided. First, the partition function is invariant under the shift of the noncommutative parameter. Second, the Euler number of the GMS soliton space on the Moyal plane is calculated and it is " 1 " for the scalar potential with even degree and "0" for odd degree tells us that the partition function of cohomological field theory is the Euler number of solution space $\mathcal{M}$ when the space is commutative. We expect that the noncommutative case is the same as the commutative case. Indeed, as we saw in the previous section, it is possible to identify the partition function of the noncommutative cohomological field theory with the Euler number as a result of the fundamental theorem of Morse theory, that is extended to the noncommutative field theory. In this paper, we saw this relation in a scalar model, but it is expected that there is no obstacle to applying general cases.

It is possible to use our method for more complex models. For example, we can estimate the Euler number of the moduli space of instantons on noncommutative $\mathbf{R}^{4}$. In that case, the partition function is the Euler number of the instanton modulispace, and there are some new moduli space of the new instanton like Nekrasov-Schwarz 21, 16). For other example, we can change the base manifold to noncommutative torus. In that case, we will have to use the Powers-Rieffel projection for calculation in the strong noncommutative limit 17,22$)$. Another point of view, we should study other types of noncommutativity. For example noncommutative parameter is locally defined type, a fuzzy sphere type and so on. To study such various cases is important to construct the local geometry of noncommutative spaces. They are left as future works.

Most of the geometric nature of the noncommutative space is still unknown. But, as we saw, it is likely that some kinds of nature of commutative space are succeeded by noncommutative space. We will have to study huge amount of them to throw light on the noncommutative geometry.

\section{Acknowledgment}

We are grateful to H.Kanno for helpful suggestions and observations and a critical reading of the manuscript. We also would like to thank H.Moriyoshi for valuable discussion. A.Sako is supported by JSPS Research Fellowships for Young Scientists.

\section{Appendix.}

\section{A Large $\theta$ limit: $\phi^{3}$ potential}

In this appendix, we show calculations in the large $\theta$ limit for simple examples of the balanced scalar model. One example is the $\phi^{3}$ potential, and another is the $\phi^{4}$ potential that is discussed in Appendix B.

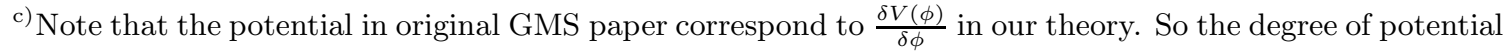
is shifted from $m$ to $m+1$.
} 
We consider the cubic potential,

$$
V(\phi)=b_{0}+b_{1} \hat{\phi}+\frac{1}{2} b_{2} \phi * \phi+\frac{1}{3 !} b_{3} \phi * \phi * \phi .
$$

The field equation is written as

$$
\begin{aligned}
\frac{\delta V}{\delta \phi} & =b_{1}+b_{2} \phi+\frac{1}{2} b_{3} \phi * \phi \\
& =\frac{1}{2} b_{3}(\phi-\alpha) *(\phi-\beta),
\end{aligned}
$$

where we put

$$
\alpha, \beta=-\left(b_{2} / b_{3}\right) \pm \sqrt{\left(b_{2} / b_{3}\right)^{2}-2\left(b_{1} / b_{3}\right)}
$$

We consider the case both $\alpha$ and $\beta$ are real numbers. By the redefinition of the field $\phi$ by a translation,

$$
\phi^{\prime} \equiv \phi-\alpha
$$

the field equation is written as

$$
\frac{\delta V}{\delta \phi}=\frac{1}{2} b_{3} \phi^{\prime} *\left(\phi^{\prime}-(\beta-\alpha)\right)=0 .
$$

The GMS solution is given by

$$
\hat{\phi}_{G M S}^{\prime}=\lambda_{i} \hat{\mathbf{P}}_{i}
$$

where the projection operator is $\hat{P}_{i}=|i\rangle\langle i|$. We put the GMS solution to the field equation,

$$
\frac{\delta V(\hat{\phi})}{\delta \hat{\phi}}=\frac{1}{2} b_{3} \lambda_{i}\left(\lambda_{i}-(\beta-\alpha)\right) \hat{\mathbf{P}}_{i}=0 .
$$

The solution is

$$
\lambda_{i}=0, \lambda_{i}=\beta-\alpha \equiv v .
$$

The general GMS solution is a linear combination of projections with coefficients $\lambda_{i}$,

$$
\hat{\phi}_{G M S}=v \sum_{i \in \mathbf{S}} \hat{\mathbf{P}}_{i}=v \hat{\mathbf{P}_{\mathbf{S}}}
$$

The bosonic part of the action is

$$
S_{B}^{\mathbf{S}}=-\left.\left.\frac{1}{4} \theta \operatorname{Tr}\left(\hat{\phi}_{q} \frac{\delta}{\delta \hat{\phi}}\right) \frac{\delta V(\hat{\phi})}{\delta \hat{\phi}}\right|_{G M S}\left(\hat{\phi}_{q} \frac{\delta}{\delta \hat{\phi}}\right) \frac{\delta V(\hat{\phi})}{\delta \hat{\phi}}\right|_{G M S} .
$$

The linear terms of expansion of $V(\hat{\phi})$ around the GMS soliton are

$$
\begin{aligned}
\left.\left(\hat{\phi}_{q} \frac{\delta}{\delta \hat{\phi}}\right) \frac{\delta V(\hat{\phi})}{\delta \hat{\phi}}\right|_{G M S} & =\left(\hat{\phi}_{q} \frac{\delta}{\delta \hat{\phi}}\right)\left[\frac{b_{3}}{2} \hat{\phi}(\hat{\phi}-v)\right]_{\phi=\phi_{G M S}} \\
& =\frac{b_{3}}{2}\left\{\hat{\phi}_{q}\left(\hat{\phi}_{G M S}-v\right)+\hat{\phi}_{G M S} \hat{\phi}_{q}\right\} \\
& =\frac{b_{3} v}{2}\left\{-\hat{\phi}_{q}\left(\hat{\mathbf{1}}-\hat{\mathbf{P}_{\mathbf{S}}}\right)+\hat{\mathbf{P}}_{\mathbf{S}} \hat{\phi}_{q}\right\} .
\end{aligned}
$$


Hence, we get

$$
S_{B}^{\mathbf{S}}=-\frac{1}{4} \theta\left(\frac{b_{3} v}{2}\right)^{2} \operatorname{Tr}\left[\hat{\phi}_{q}\left(\hat{\mathbf{1}}-\hat{\mathbf{P}}_{\mathbf{S}}\right) \hat{\phi}_{q}\left(\hat{\mathbf{1}}-\hat{\mathbf{P}}_{\mathbf{S}}\right)+\hat{\mathbf{P}}_{\mathbf{S}} \hat{\phi}_{q} \hat{\mathbf{P}}_{\mathbf{S}} \hat{\phi}_{q}\right]
$$

The fermionic part of the action is

$$
S_{F}^{\mathbf{S}}=-\left.i \theta \operatorname{Tr} \hat{\chi}\left(\hat{\psi} \frac{\delta}{\delta \hat{\phi}}\right) \frac{\delta V(\hat{\phi})}{\delta \hat{\phi}}\right|_{G M S} .
$$

As is the same as the bosonic part, the leading terms of quantum field are given by

$$
\left.\left(\hat{\psi} \frac{\delta}{\delta \hat{\phi}}\right) \frac{\delta V(\hat{\phi})}{\delta \hat{\phi}}\right|_{G M S}=\frac{b_{3} v}{2}\left\{-\hat{\psi}\left(\hat{\mathbf{1}}-\hat{\mathbf{P}_{\mathbf{S}}}\right)+\hat{\mathbf{P}_{\mathbf{S}}} \hat{\psi}\right\}
$$

Then the fermionic part of the action is written as

$$
S_{F}^{\mathbf{S}}=-\frac{\theta b_{3} v}{2} \operatorname{Tr}\left[-\hat{\chi}\left(\hat{\mathbf{1}}-\hat{\mathbf{P}}_{\mathbf{S}}\right) \hat{\psi}\left(\hat{\mathbf{1}}-\hat{\mathbf{P}}_{\mathbf{S}}\right)+\hat{\chi} \hat{\mathbf{P}}_{\mathbf{S}} \hat{\psi} \hat{\mathbf{P}}_{\mathbf{S}}\right] .
$$

The partition function is given by

$$
\begin{aligned}
\mathcal{Z}_{\mathbf{S}} & =\int \mathcal{D} \chi \mathcal{D} \psi \mathcal{D} \phi e^{-S} \\
& =\int \prod_{\substack{i>j \\
i, j \notin S}} \frac{d \phi_{i j}}{\sqrt{4 \pi \theta}} d \chi_{i j} d \psi_{i j} \prod_{\substack{i>j \\
i, j \in S}} \frac{d \phi_{i j}}{\sqrt{4 \pi \theta}} d \chi_{i j} d \psi_{i j} e^{-S_{B}^{\mathbf{S}}-S_{F}^{\mathbf{S}}} \\
& =\left[\sqrt{\left(\frac{b_{3} v}{2}\right)^{2}}\right]^{-(N-n)^{2}}\left[\sqrt{\left(\frac{b_{3} v}{2}\right)^{2}}\right]^{-n^{2}}\left[-\frac{b_{3} v}{2}\right]^{(N-n)^{2}}\left[\frac{b_{3} v}{2}\right]^{n^{2}},
\end{aligned}
$$

and we get

$$
\begin{aligned}
\mathcal{Z}_{\mathbf{S}} & =\left[-\operatorname{sgn}\left(b_{3}\right)\right]^{(N-n)^{2}}\left[\operatorname{sgn}\left(b_{3}\right)\right]^{n^{2}} \\
& =\left[-\operatorname{sgn}\left(b_{3}\right)\right]^{N-n}\left[\operatorname{sgn}\left(b_{3}\right)\right]^{n}
\end{aligned}
$$

where $n=\operatorname{rank} S$. There are $\frac{N !}{(N-n) ! n !}$ sets of the GMS solution which has the rank $n$. Then the total partition function is the sum over $\mathcal{Z}_{\mathbf{S}}$ with the weight $\frac{N !}{(N-n) ! n !}$,

$$
\begin{aligned}
\mathcal{Z}_{\text {Total }} & =\sum_{\mathbf{S}} \mathcal{Z}_{\mathbf{S}} \\
& =\lim _{N \rightarrow \infty} \sum_{n=0}^{N}{ }_{N} C_{n}\left[-\operatorname{sgn}\left(b_{3}\right)\right]^{N-n}\left[\operatorname{sgn}\left(b_{3}\right)\right]^{n}
\end{aligned}
$$

From the binomial theorem, we find that

$$
\mathcal{Z}_{\text {Total }}=0
$$

In the $\phi^{3}$ potential, the partition function is zero. 


\section{B Large $\theta$ limit: $\phi^{4}$ potential}

Here we consider another simple example which has $\phi^{4}$ potential,

$$
V(\phi)=b_{0}+b_{1} \phi+\frac{1}{2} b_{2} \phi * \phi+\frac{1}{3 !} b_{3} \phi * \phi * \phi+\frac{1}{4 !} b_{4} \phi * \phi * \phi * \phi .
$$

The field equation is always written as a factorization form

$$
\frac{\delta V}{\delta \phi}=\frac{1}{3 !} b_{4}(\phi-\alpha) *(\phi-\beta) *(\phi-\gamma) .
$$

As is the same as the $\phi^{3}$, we translate the scalar field

$$
\phi^{\prime} \equiv \phi-\alpha
$$

Then the field equation is rewritten as

$$
\begin{aligned}
\frac{\delta V}{\delta \phi} & =\frac{1}{3 !} b_{4} \phi^{\prime} *\left(\phi^{\prime}-(\beta-\alpha)\right) *\left(\phi^{\prime}-(\gamma-\alpha)\right) \\
& =\frac{1}{3 !} b_{4} \phi^{\prime} *\left(\phi^{\prime}-v_{1}\right) *\left(\phi^{\prime}-v_{2}\right),
\end{aligned}
$$

where we define

$$
v_{1} \equiv(\beta-\alpha), v_{2} \equiv(\gamma-\alpha)
$$

For simplicity we take $\alpha, \beta$ and $\gamma$ are real numbers. We put the GMS solution $\phi_{G M S}^{\prime}=\lambda_{i} \mathbf{P}_{i}$ into the field equation, then

$$
\frac{\delta V}{\delta \phi}=\frac{1}{3 !} b_{4} \lambda_{i}\left(\lambda_{i}-v_{1}\right)\left(\lambda_{i}-v_{2}\right) \mathbf{P}_{i}=0 .
$$

The solution is

$$
\lambda_{i}=0, v_{1}, v_{2}
$$

The general GMS solution is a linear combination of projections with coefficients $\lambda_{i}$,

$$
\begin{aligned}
\phi_{G M S} & =v_{1} \sum_{i \in \mathbf{S}_{\mathbf{1}}} \mathbf{P}_{i}+v_{2} \sum_{i \in \mathbf{S}_{\mathbf{2}}} \mathbf{P}_{i} \\
& =v_{1} \mathbf{P}_{\mathbf{S}_{\mathbf{1}}}+v_{2} \mathbf{P}_{\mathbf{S}_{\mathbf{2}}}
\end{aligned}
$$

The bosonic part of the action is given by

$$
S_{B}^{\mathbf{S}}=-\frac{1}{4} \operatorname{Tr}\left[\left.\left.\left(\hat{\phi}_{q} \frac{\delta}{\delta \hat{\phi}}\right) \frac{\delta V(\hat{\phi})}{\delta \hat{\phi}}\right|_{G M S}\left(\hat{\phi}_{q} \frac{\delta}{\delta \hat{\phi}}\right) \frac{\delta V(\hat{\phi})}{\delta \hat{\phi}}\right|_{G M S}\right]
$$


The linear terms of expansion of $V(\hat{\phi})$ around the GMS soliton are

$$
\begin{aligned}
\left.\left(\hat{\phi}_{q} \frac{\delta}{\delta \hat{\phi}}\right) \frac{\delta V(\hat{\phi})}{\delta \hat{\phi}}\right|_{G M S} & \left(\phi_{q} \frac{\delta}{\delta \hat{\phi}}\right)\left[\frac{b_{4}}{3 !} \hat{\phi}\left(\hat{\phi}-v_{1}\right)\left(\hat{\phi}-v_{2}\right)\right]_{\hat{\phi}=\hat{\phi}_{G M S}} \\
= & \frac{b_{4}}{3 !}\left\{\hat{\phi}_{q}\left(\hat{\phi}_{G M S}-v_{1}\right)\left(\hat{\phi}_{G M S}-v_{2}\right)\right. \\
& \left.+\hat{\phi}_{G M S} \hat{\phi}_{q}\left(\hat{\phi}_{G M S}-v_{2}\right)+\hat{\phi}_{G M S}\left(\hat{\phi}_{G M S}-v_{2}\right) \hat{\phi}_{q}\right\} \\
= & \frac{b_{4}}{3 !}\left\{\left(v_{1} v_{2}\right) \hat{\phi}_{q}\left(\hat{\mathbf{1}}-\hat{\mathbf{P}}_{\mathbf{S}_{\mathbf{1}}}-\hat{\mathbf{P}}_{\mathbf{S}_{\mathbf{2}}}\right)\right. \\
& +\left(v_{1} \hat{\mathbf{P}}_{\mathbf{S}_{\mathbf{1}}} v_{2} \hat{\mathbf{P}}_{\mathbf{S}_{\mathbf{2}}}\right) \hat{\phi}_{q} \\
& {\left[-v_{2}\left(\hat{\mathbf{1}}_{-}-\hat{\mathbf{P}}_{\mathbf{S}_{\mathbf{1}}}-\hat{\mathbf{P}}_{\mathbf{S}_{\mathbf{2}}}\right)+\left(v_{1}-v_{2}\right) \hat{\mathbf{P}}_{\mathbf{S}_{\mathbf{1}}}\right] } \\
& \left.+v_{2}\left(v_{2}-v_{1}\right) \hat{\mathbf{P}}_{\mathbf{S}_{\mathbf{2}}} \hat{\phi}_{q}\right\} .
\end{aligned}
$$

The bosonic part of the action is

$$
\begin{aligned}
S_{B}^{\mathbf{S}}=-\frac{1}{4} \theta \operatorname{Tr}[ & \left(b_{4} \zeta_{0}\right)^{2} \hat{\phi}_{q}\left(\hat{\mathbf{1}}-\hat{\mathbf{P}}_{\mathbf{S}_{\mathbf{1}}}-\hat{\mathbf{P}}_{\mathbf{S}_{\mathbf{2}}}\right) \hat{\phi}_{q}\left(\hat{\mathbf{1}}-\hat{\mathbf{P}}_{\mathbf{S}_{\mathbf{1}}}-\hat{\mathbf{P}}_{\mathbf{S}_{\mathbf{2}}}\right) \\
& \left.+\left(b_{4} \zeta_{1}\right)^{2} \hat{\mathbf{P}}_{\mathbf{S}_{\mathbf{1}}} \hat{\phi}_{q} \hat{\mathbf{P}}_{\mathbf{S}_{\mathbf{1}}} \hat{\phi}_{q}+\left(b_{4} \zeta_{2}\right) \hat{\mathbf{P}}_{\mathbf{S}_{\mathbf{2}}} \hat{\phi}_{q} \hat{\mathbf{P}}_{\mathbf{S}_{\mathbf{2}}} \hat{\phi}_{q}\right]
\end{aligned}
$$

where we define

$$
\zeta_{0} \equiv \frac{1}{3 !} v_{1} v_{2}, \zeta_{1} \equiv \frac{1}{3 !} v_{1}\left(v_{1}-v_{2}\right), \zeta_{2} \equiv \frac{1}{3 !} v_{2}\left(v_{2}-v_{1}\right) .
$$

The fermionic part of the Lagrangian is written as

$$
S_{F}^{\mathbf{S}}=-\left.i \theta \operatorname{Tr} \hat{\chi}\left(\hat{\psi} \frac{\delta}{\delta \hat{\phi}}\right) \frac{\delta V(\hat{\phi})}{\delta \hat{\phi}}\right|_{G M S} .
$$

The BRST operation is given by

$$
\begin{aligned}
\left.\left(\hat{\psi} \frac{\delta}{\delta \hat{\phi}}\right) \frac{\delta V(\hat{\phi})}{\delta \hat{\phi}}\right|_{G M S}=\frac{b_{4}}{3 !}\{ & \left(v_{1} v_{2}\right) \hat{\psi}\left(\hat{\mathbf{1}}-\hat{\mathbf{P}}_{\mathbf{S}_{\mathbf{1}}}-\hat{\mathbf{P}}_{\mathbf{S}_{\mathbf{2}}}\right) \\
& +\left(v_{1} \hat{\mathbf{P}}_{\mathbf{S}_{\mathbf{1}}}+v_{2} \hat{\mathbf{P}}_{\mathbf{S}_{\mathbf{2}}}\right) \hat{\psi} \\
& \times\left[-v_{2}\left(\hat{\mathbf{1}}-\hat{\mathbf{P}}_{\mathbf{S}_{\mathbf{1}}}-\hat{\mathbf{P}}_{\mathbf{S}_{\mathbf{2}}}\right)+\left(v_{1}-v_{2}\right) \hat{\mathbf{P}}_{\mathbf{S}_{\mathbf{1}}}\right] \\
& \left.+v_{2}\left(v_{2}-v_{1}\right) \hat{\mathbf{P}}_{\mathbf{S}_{\mathbf{2}}} \hat{\psi}\right\}
\end{aligned}
$$

Then the fermionic part is

$$
\begin{aligned}
S_{F}^{\mathbf{S}}=-i \theta \operatorname{Tr}\left[b_{4} \zeta_{0}\right. & \hat{\chi}\left(\hat{\mathbf{1}}-\hat{\mathbf{P}}_{\mathbf{S}_{\mathbf{1}}}-\hat{\mathbf{P}}_{\mathbf{S}_{\mathbf{2}}}\right) \hat{\psi}\left(\hat{\mathbf{1}}-\hat{\mathbf{P}}_{\mathbf{S}_{\mathbf{1}}}-\hat{\mathbf{P}}_{\mathbf{S}_{\mathbf{2}}}\right) \\
& +b_{4} \zeta_{1} \hat{\chi} \hat{\mathbf{P}}_{\mathbf{S}_{\mathbf{1}}} \hat{\psi} \hat{\mathbf{P}}_{\mathbf{S}_{\mathbf{1}}} \\
& \left.+b_{4} \zeta_{2} \hat{\chi} \hat{\mathbf{P}}_{\mathbf{S}_{\mathbf{2}}} \hat{\psi} \hat{\mathbf{P}}_{\mathbf{S}_{\mathbf{2}}}\right]
\end{aligned}
$$


The partition function is given

$$
\begin{aligned}
\mathcal{Z}_{\mathbf{S}}= & \int \mathcal{D} \chi \mathcal{D} \psi \mathcal{D} \phi e^{-S} \\
= & \int \prod_{\mathbf{S}=\mathbf{S}_{\mathbf{0}}}^{S_{2}}\left[\prod_{\substack{m>n \\
i, j \in \mathbf{S}}} \frac{d \phi_{i j}}{\sqrt{4 \pi \theta}} d \chi_{i j} d \psi_{i j} e^{-S_{B}^{\mathbf{S}}-S_{F}^{\mathbf{S}}}\right. \\
= & \times\left[\sqrt{\left(b_{4} \zeta_{0}\right)^{2}}\right]^{-\left(N-n_{1}-n_{2}\right)^{2}}\left[\sqrt{\left(b_{4} \zeta_{1}\right)^{2}}\right]^{-n_{1}^{2}}\left[\sqrt{\left(b_{4} \zeta_{2}\right)^{2}}\right]^{-n_{2}^{2}} \\
& \times\left[b_{4} \zeta_{0}\right]^{\left(N-n_{1}-n_{2}\right)^{2}}\left[b_{4} \zeta_{1}\right]^{n_{1}^{2}}\left[b_{4} \zeta_{2}\right]^{n_{2}^{2}} .
\end{aligned}
$$

Hence, we get

$$
\mathcal{Z}_{\mathbf{S}}=\left[\operatorname{sgn}\left(b_{4} \zeta_{0}\right)\right]^{\left(N-n_{1}-n_{2}\right)^{2}}\left[\operatorname{sgn}\left(b_{4} \zeta_{1}\right)\right]^{n_{1}^{2}}\left[\operatorname{sgn}\left(b_{4} \zeta_{2}\right)\right]^{n_{2}^{2}}
$$

where $n_{1}=\operatorname{rank} S_{1}, n_{2}=\operatorname{rank} S_{2}$. From the definition of $\zeta_{i}$ 's, one of $\operatorname{sgn}\left(\zeta_{i}\right)$ is always negative, and the others are positive. Then the partition function is always written as

$$
\begin{aligned}
\mathcal{Z}_{\mathbf{S}} & =\left[\operatorname{sgn}\left(b_{4}\right)\right]^{\left(N-n_{1}-n_{2}\right)^{2}}\left[-\operatorname{sgn}\left(b_{4}\right)\right]^{n_{1}^{2}}\left[\operatorname{sgn}\left(b_{4}\right)\right]^{n_{2}^{2}} \\
& =\left[\operatorname{sgn}\left(b_{4}\right)\right]^{N-n_{1}-n_{2}}\left[-\operatorname{sgn}\left(b_{4}\right)\right]^{n_{1}}\left[\operatorname{sgn}\left(b_{4}\right)\right]^{n_{2}}
\end{aligned}
$$

There are $\frac{N !}{\left(N-n_{1}-n_{2}\right) ! n_{1} ! n_{2} !}$ sets of the GMS solution when the rank of $\mathbf{S}_{1}$ and $\mathbf{S}_{2}$ are $n_{1}$ and $n_{2}$. Then the total partition function is the sum over $\mathbf{Z}_{S}$ with the weight $\frac{N !}{\left(N-n_{1}-n_{2}\right) ! n_{1} ! n_{2} !} \cdot$

$$
\begin{aligned}
\mathcal{Z}_{\text {Total }} & =\sum_{\mathbf{S}} \mathcal{Z}_{\mathbf{S}} \\
& =\lim _{N \rightarrow \infty} \sum_{n_{1}, n_{2}=0}^{N} \frac{N !}{\left(N-n_{1}-n_{2}\right) ! n_{1} ! n_{2} !}\left[\operatorname{sgn}\left(b_{4}\right)\right]^{N-n_{1}-n_{2}}\left[-\operatorname{sgn}\left(b_{4}\right)\right]^{n_{1}}\left[\operatorname{sgn}\left(b_{4}\right)\right]^{n_{2}} \\
& =\lim _{N \rightarrow \infty}\left(\operatorname{sgn}\left(b_{4}\right)-\operatorname{sgn}\left(b_{4}\right)+\operatorname{sgn}\left(b_{4}\right)\right)^{N} \\
& =\lim _{N \rightarrow \infty}\left(\operatorname{sgn}\left(b_{4}\right)\right)^{N} .
\end{aligned}
$$

In the $\phi^{4}$ potential, the partition function takes a non-vanishing value $\lim _{N \rightarrow \infty}\left(\operatorname{sgn}\left(b_{4}\right)\right)^{N}$.

\section{Gaussian integral in the noncommutative space}

In our calculations, the Gaussian functional integral appears, whose form is

$$
\int \mathcal{D} \phi \exp \left\{-\left.\int d^{2} x \phi(x) * V(\phi(x))\right|_{G M S} * \phi(x)\right\}
$$

and we convert it into the number representation. Generally, the difference of the operator ordering may yield some difference of the result. Therefore we must show that our prescription is correct.

Generally operators can be represented in the number representation as

$$
\hat{O}=\sum_{m, n} O_{m n}|m\rangle\langle n|
$$


where $\hat{O}$ is any operator and $|m\rangle\langle n|$ is the basis of the number representation. Any operator can be represented as the Weyl ordered operator, so the basis $|m\rangle\langle n|$ can be written as

$$
|m\rangle\langle n|=\underbrace{\int \frac{d^{2} k}{(2 \pi)^{2}} \tilde{f}_{m n}(k) e^{i\left(k_{1} \hat{x}^{1}+k_{2} \hat{x}^{2}\right)},}_{\text {Weyl ordered operator }}
$$

and its Weyl ordered symbol corresponding to the basis $|m\rangle\langle n|$ is

$$
f_{m n}(x)=\int \frac{d^{2} k}{(2 \pi)^{2}} \tilde{f}_{m n}(k) e^{i\left(k_{1} x^{1}+k_{2} x^{2}\right)} .
$$

Here, we consider the following integral,

$$
\begin{aligned}
& \int d \hat{x}_{1} d \hat{x}_{2}|m\rangle\langle n \mid p\rangle\langle q| \\
= & \int d \hat{x}_{1} d \hat{x}_{2} \int \frac{d^{2} k}{(2 \pi)^{2}} \frac{d^{2} k^{\prime}}{(2 \pi)^{2}} \tilde{f}_{m n}(k) \tilde{f}_{p q}\left(k^{\prime}\right) e^{i\left(k_{1} \hat{x}^{1}+k_{2} \hat{x}^{2}\right)} e^{i\left(k_{1}^{\prime} \hat{x}^{1}+k_{2}^{\prime} \hat{x}^{2}\right)} \\
= & \int \frac{d^{2} k}{(2 \pi)^{2}} \tilde{f}_{m n}(k) \tilde{f}_{p q}(-k),
\end{aligned}
$$

where we set $\left[x^{1}, x^{2}\right]=1$ for simplicity and use Baker-Campbell-Hausdorff formula. On the other hand, since $|m\rangle\langle n \mid p\rangle\langle q|=| m\rangle \delta_{n p}\langle q|$,

$$
\begin{aligned}
& \int d \hat{x}_{1} d \hat{x}_{2}|m\rangle \delta_{n p}\langle q| \\
= & \int d \hat{x}_{1} d \hat{x}_{2} \int \frac{d^{2} k}{(2 \pi)^{2}} \tilde{f}_{m q}(k) \delta_{n p} e^{i\left(k_{1} \hat{x}^{1}+k_{2} \hat{x}^{2}\right)} \\
= & \int d^{2} k \tilde{f}_{m q}(k) \delta^{2}(k) \delta_{n p} .
\end{aligned}
$$

Therefore we can derive a relation:

$$
\int \frac{d^{2} k}{(2 \pi)^{2}} \tilde{f}_{m n}(k) \tilde{f}_{p q}(-k)=\int d^{2} k \tilde{f}_{m q}(k) \delta^{2}(k) \delta_{n p} .
$$

Using Eq. C. 7), we get the following relation

$$
\int d^{2} x f_{m n}(x) * f_{p q}(x)=\int d^{2} x f_{m q}(x) \delta_{n p}
$$

A similar calculation gives

$$
\int d^{2} x f_{p_{1} q_{1}}(x) * f_{p_{2} q_{2}}(x) * \cdots * f_{p_{n} q_{n}}(x)=\int d^{2} x f_{p_{1} q_{n}}(x) \delta_{q_{1} p_{2}} \delta_{q_{2} p_{3}} \cdots \delta_{q_{n-1} p_{n}} .
$$

It is possible to expanded any function in terms of $f_{m n}(x)$, so $\phi(x)$ and $\left.V(\phi)\right|_{G M S}$ in Eq.(C. 1) are expanded as

$$
\begin{aligned}
\phi(x) & =\sum_{m n} \phi_{m n} f_{m n}(x), \\
\left.V(\phi)\right|_{G M S} & =\sum_{l} V_{l} f_{l l}(x) .
\end{aligned}
$$


Therefore we perform the functional integral (C. 1) using Eq.(C. 8)(C. 9) as follows,

$$
\int \mathcal{D} \phi \exp \left\{-\int d^{2} x \phi_{m n} V_{l} \phi_{p q}\left(f_{m n}(x) * f_{l l}(x) * f_{p q}\right)\right\}=\left[\operatorname{det}\left|V_{l}\right|^{\frac{1}{2}}\right]^{-1} .
$$

On the other hand, a calculation in the number representation gives

$$
\begin{aligned}
& \int \mathcal{D} \hat{\phi} \exp \left\{-\operatorname{Tr} \phi_{m n} V_{l} \phi_{p q}(|m\rangle\langle n \mid l\rangle\langle l \mid p\rangle\langle q|)\right\} \\
= & \int \mathcal{D} \phi \exp \left(-\phi_{m l} V_{l} \phi_{l m}\right) \\
= & {\left[\operatorname{det}\left|V_{l}\right|^{\frac{1}{2}}\right]^{-1}, }
\end{aligned}
$$

which is the same result as Eq.(C. 12) without normalizing constant which is canceled out. Therefore we can perform integration in both representation, and the number operator is used in our calculations.

\section{D $\quad \$_{n}=0$}

We prove a theorem here.

The theorem which we will prove here is

$$
\sum_{i=B}^{A} \prod_{\alpha=0}^{i-1}\left(v_{A}-v_{\alpha}\right) \prod_{\beta=i+1}^{r}\left(v_{B}-v_{\beta}\right)=0
$$

where integers $A, B$ satisfy $0 \leqslant B<A \leqslant r$, and $v_{i}$ is any real number. We defined the following notation formally for convenience,

$$
\prod_{\alpha=0}^{-1}\left(v_{A}-v_{\alpha}\right)=\prod_{\alpha=r+1}^{r}\left(v_{A}-v_{\alpha}\right)=1 .
$$

Its proof is as follows.

$\underline{\text { Proof }}$

$$
\begin{aligned}
& \sum_{i=B}^{A} \prod_{\alpha=0}^{i-1}\left(v_{A}-v_{\alpha}\right) \prod_{\beta=i+1}^{r}\left(v_{B}-v_{\beta}\right) \\
= & \sum_{i=B}^{A}\left(v_{A}-v_{0}\right)\left(v_{A}-v_{1}\right) \cdots\left(v_{A}-v_{i-1}\right)^{\vee}\left(v_{B}-v_{i+1}\right)\left(v_{B}-v_{i+2}\right)\left(v_{B}-v_{m}\right) \\
= & \underbrace{\left(v_{A}-v_{0}\right)\left(v_{A}-v_{1}\right) \cdots\left(v_{A}-v_{B-1}\right)}_{B} \underbrace{\left(v_{B}-v_{B+1}\right)\left(v_{B}-v_{B+2}\right) \cdots\left(v_{B}-v_{m}\right)}_{B+1} \\
& +\underbrace{\left(v_{A}-v_{0}\right)\left(v_{A}-v_{1}\right) \cdots\left(v_{A}-v_{B}\right)}_{r-B} \underbrace{\left(v_{B}-v_{B+2}\right)\left(v_{B}-v_{B+3}\right) \cdots\left(v_{B}-v_{m}\right)}_{r-B-1} \\
& +\cdots \\
& +\underbrace{\left(v_{A}-v_{0}\right)\left(v_{A}-v_{1}\right) \cdots\left(v_{A}-v_{A-1}\right)}_{A} \underbrace{\left(v_{B}-v_{A+1}\right)\left(v_{B}-v_{A+2}\right) \cdots\left(v_{B}-v_{m}\right)}_{r-A}
\end{aligned}
$$




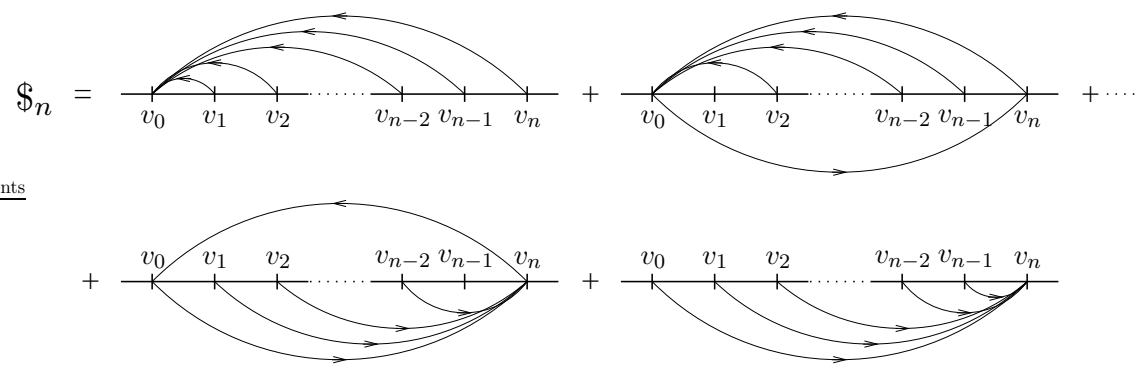

Figure 3: Graphical representation of $\$_{n}$. The arrow $v_{i} \leftarrow v_{j}$ means $\left(v_{j}-v_{i}\right)$, and all arrows in one figure should be multiplied.

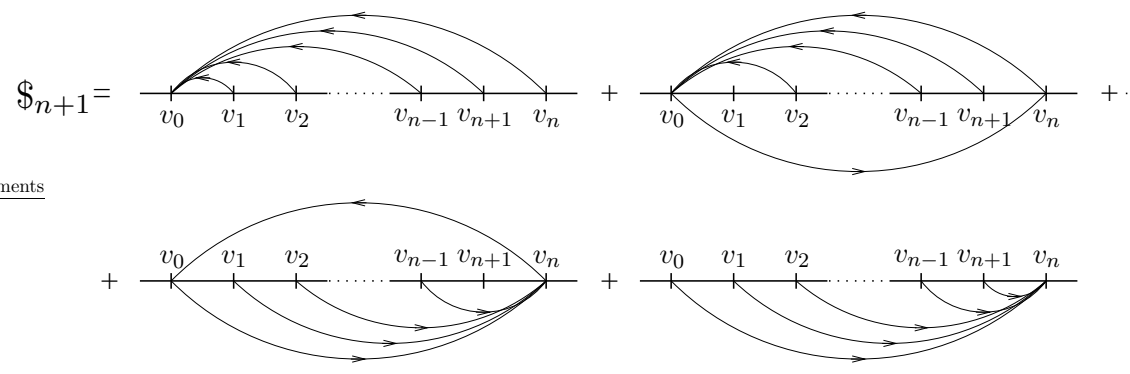

Figure 4: Graphical representation of $\$_{n+1}$. We put $v_{n+1}$ between $v_{n-1}$ and $v_{n}$ in the proof.

$$
\begin{aligned}
=\left(v_{A}-v_{0}\right)\left(v_{A}-v_{1}\right) \cdots\left(v_{A}-v_{B-1}\right) \cdot\left(v_{B}-v_{A+1}\right)\left(v_{B}-v_{A+2}\right) \cdots\left(v_{B}-v_{m}\right) \\
\times\left\{1 \cdot\left(v_{B}-v_{B+1}\right)\left(v_{B}-v_{B+2}\right) \cdots\left(v_{B}-v_{A}\right)\right. \\
\quad+\left(v_{A}-v_{B}\right) \cdot\left(v_{B}-v_{B+2}\right)\left(v_{B}-v_{B+3}\right) \cdots\left(v_{B}-v_{A}\right) \\
\quad+\cdots \\
\left.\quad+\left(v_{A}-v_{B}\right)\left(v_{A}-v_{B+1}\right) \cdots\left(v_{A}-v_{A-1}\right) \cdot 1\right\} .
\end{aligned}
$$

To show that $\{\cdots\}$ in Eq.(D. 3) equals to zero, we define $\$_{n}$ as (Fig. [3)

$$
\begin{aligned}
\$_{n}= & \left(v_{0}-v_{1}\right)\left(v_{0}-v_{2}\right)\left(v_{0}-v_{3}\right) \cdots\left(v_{0}-v_{n}\right) \\
+ & \left(v_{n}-v_{0}\right) \cdot\left(v_{0}-v_{2}\right)\left(v_{0}-v_{3}\right) \cdots\left(v_{0}-v_{n}\right) \\
& +\left(v_{n}-v_{0}\right)\left(v_{n}-v_{1}\right) \cdot\left(v_{0}-v_{3}\right) \cdots\left(v_{0}-v_{n}\right) \\
& +\cdots \\
& +\left(v_{n}-v_{0}\right) \cdots\left(v_{n}-v_{n-3}\right) \cdot\left(v_{0}-v_{n-1}\right)\left(v_{0}-v_{n}\right) \\
& +\left(v_{n}-v_{0}\right) \cdots\left(v_{n}-v_{n-3}\right)\left(v_{n}-v_{n-2}\right) \cdot\left(v_{0}-v_{n}\right) \\
& +\left(v_{n}-v_{0}\right) \cdots\left(v_{n}-v_{n-3}\right)\left(v_{n}-v_{n-2}\right)\left(v_{n}-v_{n-1}\right) .
\end{aligned}
$$

We prove $\$_{n}=0$ using mathematical induction as follows. First, for $n=1$,

$$
\$_{1}=\left(v_{0}-v_{1}\right)+\left(v_{1}-v_{0}\right)=0 .
$$


Next, we suppose $\$_{n}=0$. And we consider $\$_{n+1}$ as (Fig.⿴囗十)

$$
\begin{aligned}
\$_{n+1}= & \left(v_{0}-v_{1}\right)\left(v_{0}-v_{2}\right)\left(v_{0}-v_{3}\right) \cdots\left(v_{0}-v_{n-1}\right)\left(v_{0}-v_{n+1}\right)\left(v_{0}-v_{n}\right) \\
& +\left(v_{n}-v_{0}\right) \cdot\left(v_{0}-v_{2}\right)\left(v_{0}-v_{3}\right) \cdots\left(v_{0}-v_{n-1}\right)\left(v_{0}-v_{n+1}\right)\left(v_{0}-v_{n}\right) \\
& +\left(v_{n}-v_{0}\right)\left(v_{n}-v_{1}\right) \cdot\left(v_{0}-v_{3}\right) \cdots\left(v_{0}-v_{n-1}\right)\left(v_{0}-v_{n+1}\right)\left(v_{0}-v_{n}\right) \\
& +\cdots \\
& +\left(v_{n}-v_{0}\right) \cdots\left(v_{n}-v_{n-2}\right) \cdot\left(v_{0}-v_{n+1}\right)\left(v_{0}-v_{n}\right) \\
& +\left(v_{n}-v_{0}\right) \cdots\left(v_{n}-v_{n-2}\right)\left(v_{n}-v_{n-1}\right) \cdot\left(v_{0}-v_{n}\right) \\
& +\left(v_{n}-v_{0}\right) \cdots\left(v_{n}-v_{n-2}\right)\left(v_{n}-v_{n-1}\right)\left(v_{n}-v_{n+1}\right) \\
= & \left\{\$_{n}-\left(v_{n}-v_{0}\right)\left(v_{n}-v_{1}\right) \cdots\left(v_{n}-v_{n-1}\right)\right\}\left(v_{0}-v_{n+1}\right) \\
& +\left(v_{n}-v_{0}\right)\left(v_{n}-v_{1}\right) \cdots\left(v_{n}-v_{n-1}\right)\left\{\left(v_{0}-v_{n}\right)+\left(v_{n}-v_{n+1}\right)\right\} \\
= & \left(v_{n}-v_{0}\right)\left(v_{n}-v_{1}\right) \cdots\left(v_{n}-v_{n-1}\right)\left\{-\left(v_{0}-v_{n+1}\right)+\left(v_{0}-v_{n}\right)+\left(v_{n}-v_{n+1}\right)\right\} \\
= & 0 .
\end{aligned}
$$

Therefore $\$_{n}=0$ is valid for any $n$. Using it, $\{\cdots\}$ in Eq.(D. 3) equals to zero, so the proof is completed.

\section{References}

1) A. Armoni, R. Minasian and S. Theisen, On non-commutative $\mathcal{N}=2$ super Yang-Mills, hep-th/0102007

2) D. Birmingham, M. Blau, M. Rakowski and G. Thompson, Topological Field Theory, Phys.Rept.209(1991)129-340.

3) B. Blackadar, K-Theory for Operator Algebras, Cambridge University Press c1998.

4) M. Blau, The Mathai-Quillen Formalism and Topological Field Theory, J.Geom.Phys.11(1993)95-127, hep-th/9203026.

5) A. Connes, Noncommutative geometry, Academic Press, 1994.

6) M. Chaichian, A. Demichev and P. Prešnajder, Quantum field theory on non-commutative space-times and the persistence of ultraviolet divergences, Nucl.Phys.B567(1-2)(2000)360-390hep-th/9812180.

7) A. Connes, M.R. Douglas and A. Schwarz, Noncommutative geometry and Matrix theory: compactification on tori, JHEP 02(1998)003, hep-th/9711162.

8) S. Cordes, G. Moore and S. Ramgoolam, Lectures on 2D Yang-Mills theory, equivariant cohomology and topological field theories, Nucl.Phys.Proc.Suppl.41(1995)184-244, hep-th/9411210.

9) R.Dijkgraaf and G.Moore, Balanced Topological Field Theories, Commun.Math.Phys. 185(1997)411-440, hep-th/9608169;

J. M. F. Labastida and Carlos Lozano, Mathai-Quillen Formulation of Twisted $N=4$ Supersymmetric Gauge Theories in Four Dimensions, Nucl.Phys.B502(1997)741-790, hep-th/9702106. 
10) R. Dijkgraaf, J. Park and B. Schroers, $N=4$ Supersymmetric Yang-Mills Theory on a Kähler Surface, hep-th/9801066.

11) M. R. Douglas, N. A. Nekrasov, Noncommuatative Field Theory, submitted to Rev.Mod.Phys., hep-th/0106048.

12) J. Froehlich, O. Grandjean and A. Recknagel, Supersymmetric quantum theory and noncommutative geometry, Commun.Math.Phys. 203(1999)119-184, math-ph/9807006.

13) R. Gopakumar, M. Headrick and M. Spradlin, On Noncommutative Multi-solitons, hep-th/0103256.

14) R. Gopakumar, S. Minwalla and A. Strominger, Noncommutative Solitons, JHEP 05(2000)020, hep-th/0003160.

15) T. J. Hollowood, V. V. Khoze and G. Travaglini, Exact results in noncommutative $N=2$ supersymmetric gauge theories, JHEP 05(2001)051, hep-th/0102045.

16) T. Ishikawa, S-I. Kuroki and A. Sako, Elongated U(1) Instantons on Noncommutative $\mathbf{R}^{4}$, hep-th/0109111.

17) H. Kajima, Y. Matsuo and T. Takayanagi, Exact Tachyon Condensation on Noncommutative Torus, JHEP 0106(2001)041, hep-th/0104143.

18) C. Lozano, Duality in Topological Quantum Field Theories, doctoral thesis, hep-th/9907123.

19) J. Milnor, Morse Theory, Princeton University Press(1963).

20) N. A. Nekrasov, Trieste lectures on solitons in noncommutative gauge theories, hep-th/0011095

21) N. A. Nekrasov and A. Schwarz, Instantons on noncommutative $R^{4}$, and $(2,0)$ superconformal six dimensional theory, Commun. Math. Phys. 198(1998)689-703, hep-th/9802068.

22) M. Rieffel, Pacific J.Math. 93(1981)415-429

M. Rieffel, Projective modules over higherdimensional noncommutative tori, Canadian J.Math. 40(1988)257-338.

23) A. Sako and T. Sasaki, Euler number of Instanton Moduli Space and Seiberg-Witten Invariants, J.Math.Phys.42(2001)130-157, hep-th/0005262.

24) N. Seiberg and E. Witten, String theory and noncommutative geometry, JHEP 9909(1999)032, hep-th/9908142.

25) C. Vafa and E. Witten, A Strong coupling test of S-duality, Nucl.Phys.B431(1994)3-77, hep-th/9408074.

26) E. Witten, Topological quantum field theory, Commun.Math.Phys.117(1988)353;

E. Witten, Introduction to cohomological field theories, Int.J.Mod.Phys.A.6(1991)2273.

27) E. Witten, SuperSymmetry and Morse Theory, J.Diff.Geom.17(1982)661-692. 